PSI-PR-03-01

TTP-03-03

hep-ph/0301241

\title{
Two-loop electroweak angular-dependent logarithms at high energies
}

\author{
A. Denner ${ }^{1 \dagger}$, M. Melles ${ }^{1 \ddagger}$, And S. Pozzorini ${ }^{2 \S}$ \\ 1 Paul Scherrer Institut \\ CH-5232 Villigen PSI, Switzerland \\ ${ }^{2}$ Institut für Theoretische Teilchenphysik, Universität Karlsruhe \\ D-76128 Karlsruhe, Germany
}

\begin{abstract}
:
We present results on the two-loop leading and angular-dependent next-to-leading logarithmic virtual corrections to arbitrary processes at energies above the electroweak scale. In the "t Hooft-Feynman gauge the relevant Feynman diagrams involving soft and collinear gauge bosons $\gamma, \mathrm{Z}, \mathrm{W}^{ \pm}$coupling to external legs are evaluated in the eikonal approximation in the region where all kinematical invariants are much larger than the electroweak scale. The logarithmic mass singularities are extracted from massive multiscale loop integrals using the Sudakov method and alternatively the sector-decomposition method in the Feynman-parameter representation. The derivations are performed within the spontaneously broken phase of the electroweak theory, and the two-loop results are in agreement with the exponentiation prescriptions that have been proposed in the literature based on a symmetric $\mathrm{SU}(2) \times \mathrm{U}(1)$ theory matched with QED at the electroweak scale.
\end{abstract}

January 2003

\footnotetext{
${ }^{\dagger}$ Ansgar.Denner@psi.ch

¥Michael.Melles@psi.ch

${ }^{\S}$ pozzorin@particle.uni-karlsruhe.de
} 


\section{Introduction}

The main task of future colliders such as the LHC [1] or an $\mathrm{e}^{+} \mathrm{e}^{-}$Linear Collider (LC) [2, 3, 4, 5] will be the investigation of the origin of electroweak symmetry breaking and the exploration of the limits of the Electroweak Standard Model. In order to disentangle effects of physics beyond the Standard Model, the inclusion of QCD and electroweak radiative corrections into the theoretical predictions is crucial.

In the energy range of future colliders, i.e. at energies above the electroweak scale, $\sqrt{s} \gg M \simeq M_{\mathrm{W}} \simeq M_{\mathrm{Z}}$, the electroweak corrections are enhanced by large logarithmic contributions [6] of the type

$$
\alpha^{L} \log ^{N}\left(\frac{s}{M^{2}}\right), \quad 1 \leq N \leq 2 L
$$

These electroweak logarithmic corrections (EWLC) can be classified in a gauge-invariant way according to the powers $N$ of the logarithms of $s / M^{2}$. The leading logarithms (LL), also known as Sudakov logarithms [7], correspond to $N=2 L$, the next-to-leading logarithms (NLL) to $N=2 L-1$, etc.

The above logarithmic terms constitute the singular part of the corrections in the massless limit, $M^{2} / s \rightarrow 0$. They result either as remnants of ultraviolet singularities after parameter renormalization, or as mass singularities from soft/collinear emission of virtual or real particles off initial or final-state particles. These latter do not cancel in observables, in contrast to the well-known soft and collinear singularities observed in QCD. This is, on the one hand, due to the fact that the masses of the weak gauge bosons provide a physical cut-off, and that there is no need to include real Z-boson and W-boson bremsstrahlung. On the other hand, the Bloch-Nordsieck theorem is violated also in inclusive quantities in non-abelian gauge theories if the asymptotic states carry non-abelian charges or in spontaneously broken abelian gauge theories if mass eigenstates result from mixing of gauge eigenstates [ 8]. This leads to the appearance of LL also in inclusive quantities in such theories and thus in the electroweak Standard Model. As a consequence, the electroweak corrections can become of the order of the QCD corrections in the $\mathrm{TeV}$ energy range.

These enhanced EWLC have found quite some interest recently; for reviews we refer to Refs. [ 9, 10]. At the one-loop level the EWLC have been obtained, on the one hand, via explicit diagrammatic calculations for many $2 \rightarrow 2$ scattering processes in the Standard Model and the Minimal Supersymmetric Standard Model [ 11, 12, 13, 14, 15. On the other hand, the universality of the electroweak LL and NLL has been proven, and general results have been given for arbitrary electroweak processes that are not mass-suppressed at high energies [16, 17] and applied, for instance, to gauge-boson pair production at the LHC [18].

The typical size of the one-loop electroweak corrections from LL and NLL for a $2 \rightarrow 2$ cross section is

$$
-\frac{\alpha}{\pi s_{\mathrm{W}}^{2}} \log ^{2}\left(\frac{s}{M^{2}}\right) \simeq-26 \%, \quad+\frac{3 \alpha}{\pi s_{\mathrm{W}}^{2}} \log \left(\frac{s}{M^{2}}\right) \simeq 16 \%
$$

respectively, at $\sqrt{s}=1 \mathrm{TeV}$, with $M=M_{\mathrm{W}}$, and $1-s_{\mathrm{W}}^{2}=c_{\mathrm{W}}^{2}=M_{\mathrm{W}}^{2} / M_{\mathrm{Z}}^{2}$. The size of the corrections increases with the number of particles in the final state, and it is important to 
note that at the TeV scale, the LL and NLL have similar size and opposite sign resulting in large cancellations [14, 19].

Resummations of the EWLC have been proposed based on techniques and results known from QCD and QED. Fadin et al. [20] have resummed the LL by means of the infrared evolution equation (IREE), which describes the all-order leading-logarithmic dependence of a matrix element with respect to the transverse-momentum cut-off $\mu_{\perp}$, within a symmetric gauge theory. This equation was applied to the electroweak theory by assuming that the $\mu_{\perp}$-integration can be split into two regimes both corresponding to symmetric gauge theories. In the regime $s \geq \mu_{\perp} \geq M, \mathrm{SU}(2) \times \mathrm{U}(1)$ symmetry was used, whereas for $M \geq \mu_{\perp} \geq \lambda$ the $\mathrm{U}(1)_{\mathrm{em}}$ symmetry was assumed. Kühn et al. have applied results from QCD to resum the logarithmic corrections to massless 4-fermion processes, $\mathrm{e}^{+} \mathrm{e}^{-} \rightarrow \mathrm{ff}$, up to the NLL [19] and even to the next-to-next-to-leading logarithms (NNLL) [21]. This was done for a symmetric $\mathrm{SU}(2) \times \mathrm{U}(1)$ theory and additional electromagnetic effects were included following the IREE approach. It was found that at $1 \mathrm{TeV}$ there is no clear hierarchy between LL, NLL, and NNLL. One of us has proposed a resummation of the NLL for arbitrary processes [ 22, 23], which relies on the prescription of matching a symmetric $\mathrm{SU}(2) \times \mathrm{U}(1)$ theory with $\mathrm{QED}$ at the electroweak scale.

All these resummations amount to exponentiations of the EWLC. The approximate size of the two-loop LL and NLL resulting from the exponentiation of the one-loop corrections (1.2) for $2 \rightarrow 2$ processes at $\sqrt{s}=1 \mathrm{TeV}$ is

$$
+\frac{\alpha^{2}}{2 \pi^{2} s_{\mathrm{W}}^{4}} \log ^{4}\left(\frac{s}{M^{2}}\right) \simeq 3.5 \%, \quad-\frac{3 \alpha^{2}}{\pi^{2} s_{\mathrm{W}}^{4}} \log ^{3}\left(\frac{s}{M^{2}}\right) \simeq-4.2 \%,
$$

respectively, and it is clear that in view of the precision objectives of a LC below the per-cent level these two-loop EWLC must be under control.

All the above resummation prescriptions result from matching a symmetric $\mathrm{SU}(2) \times$ $\mathrm{U}(1)$ theory and QED at the electroweak scale, and are based on the assumption that other effects related to spontaneous symmetry breaking may be neglected at high energies. This assumption needs to be checked by explicit diagrammatic two-loop calculations based on the electroweak Lagrangian, where all relevant effects related to spontaneous symmetry breaking are taken into account. In particular, the following non-trivial aspects need to be treated with care: (i) There is a multi-scale hierarchy of masses, $M \gg m_{f \neq t} \gg \lambda$, with heavy masses $m_{\mathrm{t}} \sim M_{\mathrm{H}} \sim M$ at the electroweak scale, light-fermion masses $m_{f \neq \mathrm{t}}$, and also an infinitesimal photon mass $\lambda$, which is used as infrared regulator. As a consequence of this hierarchy, also logarithms of the large ratios $M / m_{f \neq \mathrm{t}}$ and $m_{f \neq \mathrm{t}} / \lambda$ have to be taken into account, and the general form of logarithmic terms of order $N$ in (1.1) becomes $\log ^{N_{1}}\left(s / M^{2}\right) \log ^{N_{2}}\left(M^{2} / m_{f \neq \mathrm{t}}^{2}\right) \log ^{N_{3}}\left(m_{f \neq \mathrm{t}}^{2} / \lambda^{2}\right)$, with $N=N_{1}+N_{2}+N_{3}$. (ii) In the gaugeboson sector, the gauge-group eigenstates $B, W^{3}$ mix resulting in mass eigenstates $\gamma, \mathrm{Z}$ with a large mass gap $\lambda \ll M$. (iii) Longitudinal gauge bosons appear as physical asymptotic states.

The resummation of the LL has been checked for the massless fermionic singlet form factor in Refs. [ 24, 25] and for arbitrary processes in the Coulomb gauge in Ref. [ 26]. The resummation of the next-to-leading logarithms has so far not been confirmed by explicit 
two-loop calculations. In the present paper, we consider a specific gauge-invariant subset of the next-to-leading EWLC to exclusive processes: the angular-dependent NLL of type

$$
\alpha^{L} \log ^{2 L-1}\left(\frac{s}{M^{2}}\right) \log \left(\frac{|r|}{s}\right),
$$

where $r$ represents a generic kinematic invariant different from $s$, and the ratio $r / s$ depends on the angles between external momenta. These angular-dependent NLL are numerically important as has been stressed in Refs. [14, 19, 21]. Prescriptions for their resummation have been given in Refs. [19, 21] for massless 4-fermion processes and extended to arbitrary processes in Ref. [23]. As we have mentioned above, these prescriptions are based on symmetric gauge theories. The purpose of this paper is to check them with an explicit two-loop calculation within the spontaneously broken electroweak theory.

At one-loop order, in the 't Hooft-Feynman gauge, the angular-dependent NLL result only from diagrams where a gauge boson is exchanged between two external lines. Similarly, the angular-dependent NLL at two-loop order can be traced back to a relatively small set of Feynman diagrams. This allows us to present a diagrammatic calculation of the two-loop angular-dependent NLL for arbitrary processes. The calculation is based on the eikonal approximation. The relevant massive two-loop integrals are evaluated analytically using two independent methods, one goes back to Sudakov [7], the other uses sector decomposition of Feynman-parameter integrals [27, 28, 29].

The paper is organized as follows: In Sect. 2 we define our conventions and the approximations used in the high-energy limit and we discuss the Feynman diagrams that give rise to the leading mass singularities and the eikonal approximation. Section 3 is devoted to the description of the calculation of the two-loop integrals. The results for the contributing diagrams and their sum are presented in Sect. 4. The appendices provide information on our conventions and the explicit results of the individual loop integrals as well as relations between them.

\section{High-energy logarithmic approximation}

\subsection{Preliminaries}

We consider generic electroweak processes involving $n$ arbitrary polarized particles, which may be light or heavy chiral fermions, transverse or longitudinal gauge bosons, or Higgs bosons. As a convention, we consider $n \rightarrow 0$ processes,

$$
\varphi_{i_{1}}\left(p_{1}\right) \ldots \varphi_{i_{n}}\left(p_{n}\right) \rightarrow 0
$$

where all particles $\varphi_{i_{k}}$ and their momenta $p_{k}$ are assumed to be incoming. Corresponding $2 \rightarrow n-2$ processes are easily obtained by crossing symmetry. Our calculations are performed in the physical basis, where the external particles $\varphi_{i_{k}}$ as well as the virtual particles in the loops correspond to mass eigenstates, and mixing effects are properly taken into account. The matrix elements for the processes (2.1) and the external-leg gauge couplings are denoted with the shorthands

$$
\mathcal{M}^{i_{1} \ldots i_{n}} \equiv \mathcal{M}^{\varphi_{i_{1}} \ldots \varphi_{i_{n}}}\left(p_{1}, \ldots, p_{n}\right), \quad I_{i_{k}^{\prime} i_{k}}^{a} \equiv I_{\varphi_{i_{k}^{\prime}} \varphi_{i_{k}}}^{V^{a}}
$$


where $I_{i^{\prime} i}^{a}$ corresponds to the coupling of the gauge bosons $V^{a}=\gamma, \mathrm{Z}, \mathrm{W}^{ \pm}$to an incoming particle $\varphi_{i}$ and an outgoing particle $\varphi_{i^{\prime}}$. More details concerning gauge couplings can be found in App. A

All external-leg momenta are assumed to be on shell, $p_{k}^{2}=m_{k}^{2}$, and we restrict ourselves to the high-energy region where $s=\left(p_{1}+p_{2}\right)^{2} \approx 2 p_{1} p_{2}$ as well as all other kinematical invariants are much larger than the electroweak scale. In particular, we assume the following hierarchy of energy and mass scales ${ }^{1}$

$$
\left|\left(p_{k}+p_{l}\right)^{2}\right| \simeq\left|2 p_{k} p_{l}\right| \gg M^{2} \simeq M_{\mathrm{Z}}^{2} \simeq M_{\mathrm{W}}^{2} \gg m_{f \neq \mathrm{t}}^{2} \gg M_{\gamma}^{2} \equiv \lambda^{2} .
$$

The mass scale $M$ is used to denote a generic weak-boson mass in the logarithms, and we neglect logarithms of the ratio $M_{\mathrm{W}} / M_{\mathrm{Z}}$, which originate from the difference between the $\mathrm{Z}$ - and the W-boson mass. With $m_{f \neq t}$ we denote the masses of the light fermions. The infinitesimal photon mass $\lambda$ is used to regularize infrared divergences.

All masses of real and virtual particles are assumed to be at or below the electroweak scale. Nevertheless, our results also apply to processes involving particles with masses that are heavier but of the same order as $M$, i.e. light Higgs bosons or top quarks with $M_{\mathrm{H}} \simeq m_{\mathrm{t}} \simeq M$. However, the logarithms involving the ratios $M_{\mathrm{H}} / M$ and $m_{\mathrm{t}} / M$ are neglected.

Our next-to-leading logarithmic angular-dependent $\left(\mathrm{NLL}_{\mathrm{a}}\right)$ approximation is defined as follows. We consider corrections that are logarithmically divergent in the limit where the ratios $M / \sqrt{s}, m_{f \neq t} / M$, and $\lambda / m_{f \neq \mathrm{t}}$ vanish. From these mass-singular logarithms we retain only the leading and the next-to-leading angular-dependent ones, i.e. at $L$ loops (with $L=1,2$ ) we consider only contributions of the order

$$
\begin{aligned}
& \mathcal{O}\left[\alpha^{L} \log ^{2 L-N}\left(\frac{s}{M^{2}}\right) \prod_{i=1}^{N} \log \left(\frac{M^{2}}{m_{\text {light }, i}^{2}}\right)\right], \quad 0 \leq N \leq 2 L, \quad \text { and } \\
& \mathcal{O}\left[\alpha^{L} \log \left(\frac{\left|2 p_{k} p_{l}\right|}{s}\right) \log ^{2 L-N-1}\left(\frac{s}{M^{2}}\right) \prod_{i=1}^{N} \log \left(\frac{M^{2}}{m_{\text {light }, i}^{2}}\right)\right], \quad 0 \leq N \leq 2 L-1,
\end{aligned}
$$

where $m_{\mathrm{light}, i}$ are either light-fermion or photon masses. As stated above, the logarithms of ratios of heavy masses are neglected, i.e. we consider $\log M_{\mathrm{Z}} \simeq \log M_{\mathrm{W}} \simeq \log M_{\mathrm{H}} \simeq \log m_{\mathrm{t}}$.

In our approximation all terms that are suppressed by factors $M / \sqrt{s}, m_{f \neq \mathrm{t}} / M$ or $\lambda / m_{f \neq t}$ are neglected. In practice, all mass terms in the numerators of loop integrals are omitted in the calculations. In order to avoid factors of order $\sqrt{s} / M$ from longitudinal polarization vectors that would enhance mass-suppressed contributions, the GoldstoneBoson Equivalence Theorem (GBET) [ [30] has to be used for matrix elements involving longitudinal gauge bosons. For our purposes we can use the GBET in its naive lowestorder form since the quantum corrections to the GBET involve only two-point functions, which give no contribution in the considered $\mathrm{NLL}_{\mathrm{a}}$ order (2.4) in the 't Hooft-Feynman gauge. In practice each longitudinal gauge boson $V_{\mathrm{L}}^{a}=\mathrm{W}_{\mathrm{L}}^{ \pm}, \mathrm{Z}_{\mathrm{L}}$ has to be substituted by a corresponding would-be Goldstone boson $\Phi_{a}=\phi^{ \pm}, \chi$ using

$$
\mathcal{M}^{\varphi_{i_{1}} \ldots V_{\mathrm{L}}^{a} \ldots \varphi_{i_{n}}}=\mathrm{i}^{\left(1-Q_{\left.V^{a}\right)}\right)} \mathcal{M}^{\varphi_{i_{1}} \ldots \Phi_{a} \ldots \varphi_{i_{n}}},
$$

\footnotetext{
${ }^{1}$ The first inequality implies, in particular, that all angles are larger than $M / \sqrt{s}$ in reference frames where all particle energies are not much larger than $\sqrt{s}$.
} 
where $Q_{V^{a}}= \pm 1,0$ is the corresponding charge. Thus, the general results for EWLC presented in the following have to be applied to the matrix elements involving would-beGoldstone bosons.

We restrict ourselves to matrix elements that are not mass-suppressed in the highenergy limit. This permits us to make use of the identity ${ }^{2}$

$$
\sum_{k=1}^{n} \mathcal{M}^{i_{1} \ldots i_{k}^{\prime} \ldots i_{n}} I_{i_{k}^{\prime} i_{k}}^{a}=\mathcal{O}\left(\frac{M}{\sqrt{s}}\right) \mathcal{M}^{i_{1} \ldots i_{n}} \simeq 0,
$$

which can be derived from global $\mathrm{SU}(2) \times \mathrm{U}(1)$ symmetry and is very useful in order to simplify sums over external-leg insertions of the gauge-group generators.

\subsection{Feynman diagrams in eikonal approximation}

Mass singularities originate from diagrams with virtual particles coupling to on-shell external legs. In this paper we consider only $\mathrm{NLL}_{\mathrm{a}}$ contributions that result from the leading mass singularities, i.e. at two loops from contributions involving four mass-singular logarithms. We perform the calculation in the 't Hooft-Feynman gauge, where the gaugeboson propagators have the same pole structure as scalar propagators and the leading mass singularities originate only from diagrams with soft-collinear virtual gauge bosons coupling to different external particles. The exchange of soft-collinear scalar particles or fermions is mass-suppressed. The relevant two-loop diagrams have the structure

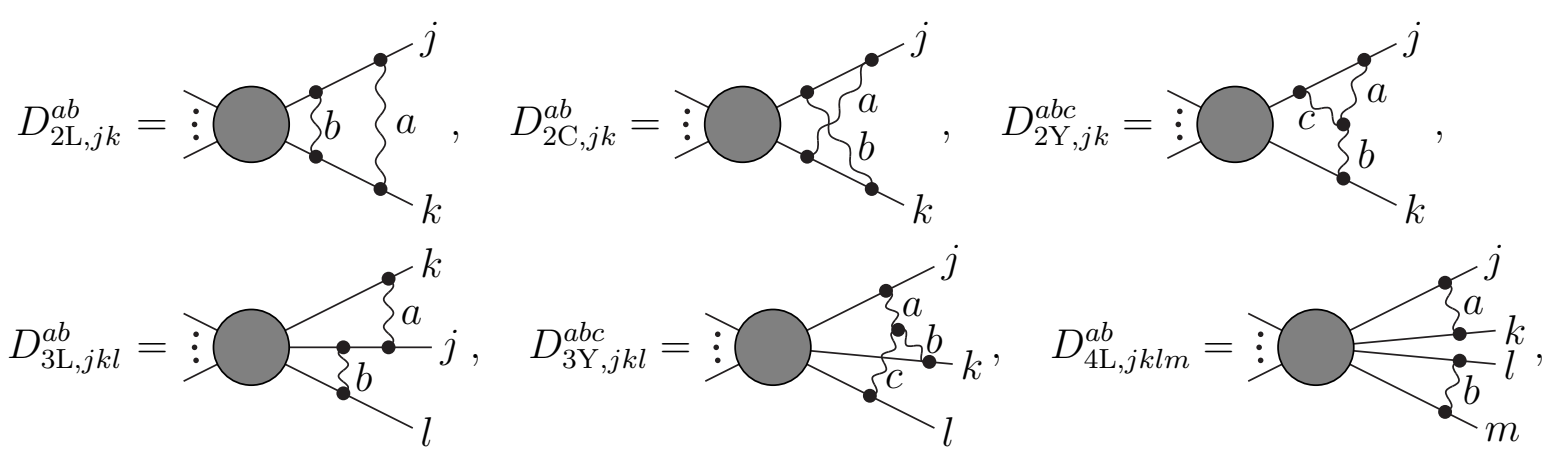

where the soft-collinear gauge bosons $V^{a}, V^{b}, V^{c}=\gamma, \mathrm{Z}, \mathrm{W}^{ \pm}$are exchanged between two, three, or four of the $n$ on-shell external legs $j, k, l, m=1, \ldots, n$. The external lines, which are generically represented by full lines, can be fermions, transverse gauge bosons, wouldbe Goldstone bosons, or Higgs bosons. The external legs that do not couple to the soft gauge bosons are represented by the lines and the dots on the left-hand side of the grey blobs.

In order to extract the leading mass singularities, the Feynman diagrams (2.7) are evaluated in eikonal approximation, i.e. by approximating the integrand in the limit where the momenta of the soft gauge bosons are small. In this limit, the above Feynman diagrams can be treated independently of the process and the spin of the external particles, i.e. universally for chiral fermions, transverse gauge bosons, or scalar particles. This is done as follows:

\footnotetext{
${ }^{2}$ Here the sums over the components $i_{k}^{\prime}$ of the multiplets corresponding to the various external particles $i_{k}$ are implicitly understood.
} 
- In the "hard part" of the diagrams corresponding to the grey blobs in (2.7) the momenta of the soft gauge bosons are neglected, and only the remaining "soft part" of the diagrams has to be integrated over the loop momenta. The blobs typically involve contributions from various tree diagrams, but they do not need to be evaluated explicitly. In our derivations we only make use of the charge-conservation identity (2.6) to relate the complete tree-level amplitudes from different blobs.

- The vertices involving three soft gauge bosons are associated with the usual YangMills couplings

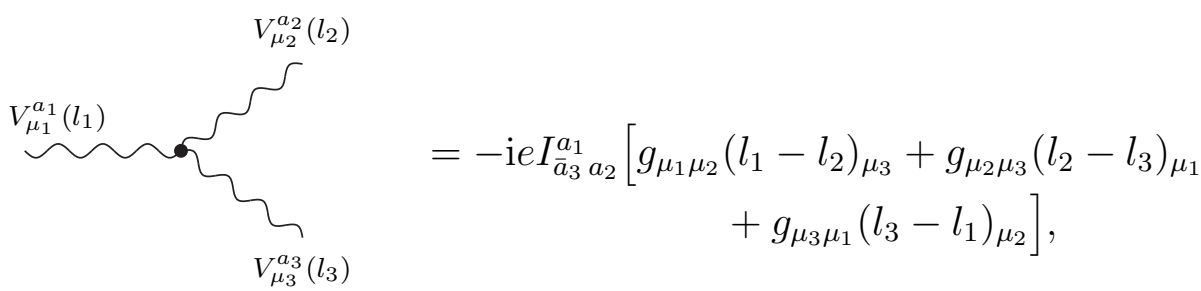

where the particles and momenta are incoming, $I_{\bar{a}_{3} a_{2}}^{a_{1}}$ are the structure constants defined by (A.3), and $\bar{a}_{i}$ indicates the complex conjugate of $a_{i}$. In the 't HooftFeynman gauge, the propagators read $-\mathrm{i} g^{\mu \nu} /\left(l^{2}-M_{a}^{2}+\mathrm{i} \varepsilon\right)$ for a gauge boson $V^{a}$.

- The emission of gauge bosons with momenta $l_{1}, l_{2}, \ldots$ along an incoming external line with momentum $k_{1}=p_{\text {ext }}$ in the soft limit $l_{i} \rightarrow 0$,

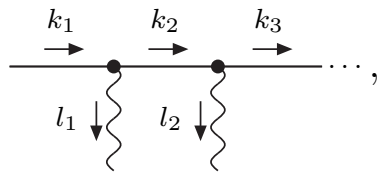

gives rise to a product of terms containing a factor $\mathrm{i} /\left(k_{j}^{2}-m_{j}^{2}+\mathrm{i} \varepsilon\right)$ for each propagator with momentum $k_{j}=k_{j-1}-l_{j-1}$ and mass $m_{j}$ and an eikonal factor [17]

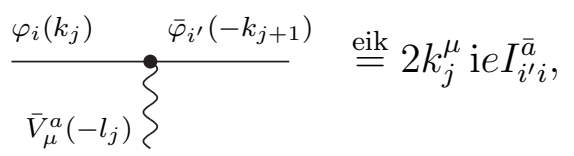

for each vertex, where $I_{i^{\prime} i}^{a}$ are the generators defined in App. A. Note that the form of these eikonal factors only depends on the gauge-group representation of the inflowing particles, but not on their spin. With $\stackrel{\text { eik }}{=}$ we denote equations that are valid within the eikonal approximation.

- The eikonal factors defined above are proportional to the momenta of the emitting particles, and if these particles are virtual they depend on the loop momentum $l_{1}$ via $k_{2}=p_{\text {ext }}-l_{1}$. If the integration is restricted to the region of soft gauge-boson momenta, $l_{1} \approx 0$, as in the Sudakov method, this loop-momentum dependence can be neglected, and one can use $k_{2}=p_{\text {ext }}$ in the eikonal factors. However, if 
one integrates over the full loop-momentum space, as in the Feynman-parameter representation, also the region $k_{2}=p_{\text {ext }}-l_{1} \approx 0$ where the emitting line becomes soft may be important. This happens for the diagrams $D_{2 \mathrm{C}}$ and $D_{3 \mathrm{~L}}$, where such regions give rise to spurious leading logarithms if one uses eikonal factors with $k_{2}=p_{\text {ext }}$, whereas the loop-momentum-dependent eikonal factors defined in (2.10) suppress them ${ }^{3}$. The reason why these contributions have to be suppressed is the following: if the emitting line in (2.9) is a fermion or a scalar particle the mass singularity for $k_{2}=0$ gets mass-suppressed in the complete Feynman diagram by the numerator. If the emitting line is a transverse gauge boson this remains true for the region $k_{2} \approx l_{2} \approx 0$, whereas the region with $k_{2} \approx 0$ and $l_{2} \approx p_{\text {ext }}^{\prime} \neq p_{\text {ext }}$, which gives leading contributions only to the diagram $D_{2 \mathrm{C}}$, has to be suppressed in order to avoid double counting of topologically equivalent configurations when the sum over all soft-collinear gauge bosons is performed.

- The denominators of the propagators denoted by full lines in (2.7) are kept exact, i.e. the square of the momenta of the soft gauge bosons is not neglected there.

The explicit expressions for the diagrams (2.7) in eikonal approximation are given in App. B.2.

All terms involving four mass-singular logarithms originate from the diagrams (2.7) and there only from the terms that are kept in the eikonal approximation. Other contributions give rise to at most three mass-singular logarithms. In this paper we assume that all angular-dependent NLL result only from contributions with four mass-singular logarithms via the appearance of different scales in the logarithms. This is equivalent to the assumption that generic NLL are not multiplied by logarithms of ratios of kinematical variables that do not result from mass singularities. Although we have not proven this assumption so far, we do not see a source for additional angular-dependent NLL. We have checked for several examples that no such terms arise from neglected contributions to the diagrams (2.7), i.e. terms with gauge-boson momenta in the numerators. A complete proof of the assumption, however, requires a calculation of the complete set of NLL.

\subsection{Mass-gap effects}

Each topology in (2.7) has to be evaluated for all different mass assignments corresponding to the various electroweak gauge bosons $V^{a}, V^{b}, V^{c}=\gamma, \mathrm{Z}, \mathrm{W}^{ \pm}$. In practice, for each diagram involving two or three soft-collinear gauge bosons we have the following four cases

$$
\begin{aligned}
\left(M_{a}, M_{b}\right) & =(\lambda, \lambda),(\lambda, M),(M, \lambda),(M, M), \\
\left(M_{a}, M_{b}, M_{c}\right) & =(M, M, M),(\lambda, M, M),(M, \lambda, M),(M, M, \lambda),
\end{aligned}
$$

whereas the external lines are assumed to have arbitrary masses $m_{k}^{2}$ at or below the electroweak scale.

Our main aim is to investigate the effects related to symmetry breaking, and in particular the effects of the large mass gap $\lambda \ll M$ in the gauge sector. This gives rise

\footnotetext{
${ }^{3}$ For all other topologies the loop-momentum dependence of the eikonal factors is irrelevant in NLL $_{a}$ approximation.
} 
to logarithms of the photon mass $\lambda$ and light-fermion masses that violate $\mathrm{SU}(2) \times \mathrm{U}(1)$ symmetry, such that the full electroweak result is only symmetric with respect to the unbroken $\mathrm{U}(1)_{\text {em }}$ group. Nevertheless, according to the physical picture proposed in the IREE approach [ 20] and generalized by other authors [ 19, 21, 22, 23, the EWLC are expected to exhibit a higher degree of symmetry. In this picture, the complete electroweak result factorizes into a part which exhibits $\mathrm{SU}(2) \times \mathrm{U}(1)$ symmetry and corresponds to the full electroweak result for the case $\lambda=M$, and a remaining part that originates from the mass gap $\lambda \ll M$ and exhibits $\mathrm{U}(1)_{\mathrm{em}}$ symmetry.

In order to check this picture at the level of angular-dependent NLL, we organize our calculation as follows. All intermediate results $f(\lambda)$ depending on the photon mass $\lambda$ are split into two parts as

$$
f(\lambda) \equiv f(M)+\Delta f(\lambda),
$$

where the part $f(M)$ corresponds to the case $\lambda=M$ and has to be calculated for ${ }^{4}$

$$
\lambda=M \gg m_{f \neq t} .
$$

In this case, all mass singularities are regulated by $M$ and the light fermion masses below the electroweak scale $m_{f \neq \mathrm{t}} \ll M$ can be neglected. The remaining part, $\Delta f(\lambda)=$ $f(\lambda)-f(M)$, originates from the mass gap $\lambda \ll M$. In the language of the IREE, this subtracted part can be understood as the part of the photonic contribution that originates from below the electroweak scale.

\subsection{Validity of our results for extensions of the electroweak theory}

Our derivations depend only on a few general features of the Electroweak Standard Model, such as the underlying global gauge symmetry, the spectrum of gauge bosons, and the fact that all particle masses are of the order of the electroweak scale or lighter. All leading and next-to-leading angular-dependent logarithms originate only from (softcollinear) gauge bosons and depend only on gauge couplings. Therefore our results apply also to those extensions of the Electroweak Standard Model, where these features are preserved, i.e. where no additional gauge bosons and no logarithms involving mass scales much higher than the electroweak scale appear.

Such models include softly broken supersymmetric extensions such as the Minimal Supersymmetric Standard Model in the case where the masses of the superpartner particles are of the order of the electroweak scale $M$. Owing to mixing, the gauge couplings $I_{i^{\prime} i}^{a}$ may involve mixing matrices. More details about higher-order supersymmetric EWLC can be found in Ref. [31].

\section{Loop integrals in logarithmic approximation}

The loop integrals were evaluated in logarithmic approximation using two independent methods: the Sudakov technique and the sector-decomposition method described in Sect. 3.1 and Sect. 3.2, respectively. These two methods were applied in a complementary way in order to calculate all integrals in App. B and to perform various cross checks.

\footnotetext{
${ }^{4}$ Note that this part $f(M)$ cannot be obtained by simply substituting $\lambda=M$ in the result $f(\lambda)$ since the inequalities (2.3) and (2.14) exclude each other.
} 


\subsection{The Sudakov technique for angular-dependent logarithms}

The Sudakov technique has long been known and used for the calculation of the leading logarithmic asymptotics of field theories [ 17, 32. In this paper, we apply this approach to the calculation of the leading and next-to-leading angular-dependent logarithms at the two-loop level. To our knowledge, the Sudakov technique has so far only been applied to ladder or crossed ladder diagrams involving a single large invariant. Here we generalize this method to the case of different large invariants.

We illustrate the method for the 3-leg ladder diagram $D_{3 \mathrm{~L}, j k l}^{a b}$ shown in (B.21). In the eikonal approximation the corresponding integral is given by (B.22). It involves four different mass singularities and thus gives rise to four large logarithms. These singularities appear if the gauge-boson momenta $l_{1}$ and $l_{2}$ become soft and collinear to the momenta of the external particles to which the gauge bosons couple. Mass terms have to be only retained as far as they regularize the mass singularities.

In order to extract these singularities it is convenient to use the following Sudakov parametrizations for the loop momenta,

$$
\begin{aligned}
& l_{1}=y_{1}\left(p_{j}-\frac{m_{j}^{2}}{2 p_{j} p_{k}} p_{k}\right)+x_{1}\left(p_{k}-\frac{m_{k}^{2}}{2 p_{j} p_{k}} p_{j}\right)+l_{1, \perp}, \\
& l_{2}=y_{2}\left(p_{j}-\frac{m_{j}^{2}}{2 p_{j} p_{l}} p_{l}\right)+x_{2}\left(p_{l}-\frac{m_{l}^{2}}{2 p_{j} p_{l}} p_{j}\right)+l_{2, \perp}
\end{aligned}
$$

where $l_{1, \perp} p_{j}=l_{1, \perp} p_{k}=l_{2, \perp} p_{j}=l_{2, \perp} p_{l}=0$. The mass terms turn out to be only relevant for photon exchange since we assume $m_{j, k, l} \lesssim M$. The two-dimensional transverse momenta $l_{i, \perp}$ are space-like. They can be parametrized by their moduli $\left|\mathbf{l}_{i, \perp}\right|$ and azimuthal angles $\phi_{i}$. Then up to irrelevant mass terms, the integration measures read $\mathrm{d}^{4} l_{1}=\frac{1}{2}\left|p_{j} p_{k}\right| \mathrm{d} \mathbf{l}_{1, \perp}^{2} \mathrm{~d} \phi_{1} \mathrm{~d} x_{1} \mathrm{~d} y_{1}$ and $\mathrm{d}^{4} l_{2}=\frac{1}{2}\left|p_{j} p_{l}\right| \mathrm{d} \mathbf{l}_{2, \perp}^{2} \mathrm{~d} \phi_{2} \mathrm{~d} x_{2} \mathrm{~d} y_{2}$.

Since the leading logarithms originate from the regime of soft and collinear gaugeboson momenta, we can drop all $l$-dependent terms in the numerator and replace the gauge-boson propagators as

$$
\begin{aligned}
& \frac{\mathrm{i}}{l_{1}^{2}-M_{a}^{2}+\mathrm{i} \varepsilon}=\frac{\mathrm{i}}{2 p_{j} p_{k} x_{1} y_{1}-M_{a}^{2}-\mathrm{l}_{1, \perp}^{2}+\mathrm{i} \varepsilon} \rightarrow \pi \delta\left(2 p_{j} p_{k} x_{1} y_{1}-M_{a}^{2}-\mathrm{l}_{1, \perp}^{2}\right), \\
& \frac{\mathrm{i}}{l_{2}^{2}-M_{b}^{2}+\mathrm{i} \varepsilon}=\frac{\mathrm{i}}{2 p_{j} p_{l} x_{2} y_{2}-M_{b}^{2}-\mathrm{l}_{2, \perp}^{2}+\mathrm{i} \varepsilon} \rightarrow \pi \delta\left(2 p_{j} p_{l} x_{2} y_{2}-M_{b}^{2}-\mathrm{l}_{2, \perp}^{2}\right),
\end{aligned}
$$

up to irrelevant terms of order $m_{j, k, l}^{4}$. Performing the integrals over $\mathbf{l}_{i, \perp}^{2}$ with the help of the $\delta$ functions, we find after neglecting irrelevant mass terms

$$
\begin{aligned}
& S_{3 \mathrm{~L}}\left(M_{a}, M_{b} ; p_{j}, p_{k}, p_{l}\right)=\frac{4}{\pi^{2}}\left(p_{j} p_{k}\right)\left(p_{j} p_{l}\right)\left|p_{j} p_{k}\right|\left|p_{j} p_{l}\right| \int \mathrm{d} x_{1} \mathrm{~d} y_{1} \mathrm{~d} x_{2} \mathrm{~d} y_{2} \mathrm{~d} \phi_{1} \mathrm{~d} \phi_{2} \\
& \times \theta\left(2 p_{j} p_{k} x_{1} y_{1}-M_{a}^{2}\right) \theta\left(2 p_{j} p_{l} x_{2} y_{2}-M_{b}^{2}\right) \\
& \times\left[2 p_{j} p_{k} x_{1}+m_{j}^{2} y_{1}+M_{a}^{2}\right]^{-1}\left[-2 p_{j} p_{k} y_{1}-m_{k}^{2} x_{1}+M_{a}^{2}\right]^{-1} \\
& \times\left[2 p_{j} p_{k} x_{1}+2 p_{j} p_{l} x_{2}+m_{j}^{2}\left(y_{1}+y_{2}\right)+M_{a}^{2}+M_{b}^{2}+2 p_{k} p_{l} x_{1} x_{2}\right. \\
& \left.+2 p_{j} p_{l} x_{2} y_{1}+2 p_{j} p_{k} x_{1} y_{2}+2 \mathbf{l}_{1, \perp} p_{l} x_{2}+2 \mathbf{l}_{2, \perp} p_{k} x_{1}+2 \mathbf{l}_{1, \perp} \mathbf{l}_{2, \perp}\right]^{-1} \\
& \times\left[-2 p_{j} p_{l} y_{2}-m_{l}^{2} x_{2}+M_{b}^{2}\right]^{-1} \text {, }
\end{aligned}
$$


where $\mathrm{l}_{1, \perp}^{2}=2 x_{1} y_{1} p_{j} p_{k}-M_{a}^{2}$ and $\mathrm{l}_{2, \perp}^{2}=2 x_{2} y_{2} p_{j} p_{l}-M_{b}^{2}$ and the dependence on $\phi_{i}$ enters via $\mathbf{l}_{i, \perp}$. Terms involving four logarithms result from those parts of the integration region where all Sudakov variables are small, $\left|x_{1}\right|,\left|y_{1}\right|,\left|x_{2}\right|,\left|y_{2}\right| \ll 1$, and the integrand behaves as $1 /\left(x_{1} y_{1} x_{2} y_{2}\right)$, i.e. where each of the four denominators is dominated by a different term linear in one of the Sudakov variables. These parts of the integration region are selected by conditions of the form $\left|2 p_{j} p_{k} x_{1}\right| \gg\left|m_{j}^{2} y_{1}\right|,\left|2 p_{j} p_{l} x_{2}\right| \gg\left|2 p_{j} p_{k} x_{1}\right|$, etc. To leading-logarithmic accuracy, these conditions are implemented via step functions $\theta\left(\left|2 p_{j} p_{k} x_{1}\right|-\left|m_{j}^{2} y_{1}\right|\right), \theta\left(\left|2 p_{j} p_{l} x_{2}\right|-\left|2 p_{j} p_{k} x_{1}\right|\right)$, etc., which, in particular, ensure that none of the Sudakov variables can become zero. Upper integration limits are set to one, i.e. $\left|x_{1}\right|,\left|y_{1}\right|,\left|x_{2}\right|,\left|y_{2}\right|<1$. Then irrelevant terms are neglected in the denominators and in the step functions. Since, in particular, all terms depending on $\mathbf{l}_{i, \perp}$ and thus on $\phi_{i}$ are negligible, the integration over these angles can be performed trivially. After transforming regions with negative Sudakov variables to those with positive Sudakov variables one finally obtains (B.23).

In this approach it is crucial that the perpendicular components of the loop momenta $\mathbf{l}_{i, \perp}$ can be dropped. This is the case for all ladder diagrams, if the Sudakov parametrizations are constructed from the external on-shell momenta of the lines to which the gauge bosons couple as in (3.1).

The Yang-Mills diagram $S_{2 Y}$ with two external lines contains three virtual gauge bosons that can become soft and collinear. If any two of them go on-shell, this results in four large logarithms. Thus, one has to sum over the three contributions with different pairs of on-shell gauge bosons to obtain the full leading-logarithmic result. Since there are only two relevant external momenta, the Sudakov parametrization is unique for each of the three cases. A slight complication arises from the fact that the numerator is linear in the loop momenta. Nevertheless, one can show that all terms involving transverse loop momenta can be neglected in the leading-logarithmic approximation and the leadinglogarithmic contributions can be extracted in a way similar to the ladder diagrams.

For the non-abelian graph $S_{3 \mathrm{Y}}$ with three external lines no parametrization exists that would allow to neglect all $\mathbf{l}_{i, \perp}$ terms. For this diagram we therefore did not apply the Sudakov method but have checked the result from the sector-decomposition approach by a numerical integration of the Feynman-parameter integral.

\subsection{Sector-decomposition method}

The loop integrals were also evaluated in the Feynman-parameter representation. In this case, in order to extract the logarithmic mass singularities we used the sector decomposition [ 27, 28, 29], which permits to factorize overlapping ultraviolet or mass singularities in Feynman-parameter integrals. A detailed description of this method is postponed to a forthcoming publication [33. Here we only sketch the main steps of the sector-decomposition method applied to a generic two-loop massive integral with $n+1$ propagators

$$
\int \frac{\mathrm{d}^{d} l_{1}}{(2 \pi)^{d}} \int \frac{\mathrm{d}^{d} l_{2}}{(2 \pi)^{d}} \frac{N\left(\left\{l_{j}\right\},\left\{p_{l}\right\}\right)}{\prod_{i=1}^{n+1}\left(k_{i}^{2}-m_{i}^{2}+\mathrm{i} \varepsilon\right)},
$$

where the momenta $k_{i}$ are linear combinations of the external momenta $p_{l}$ and the loop momenta $l_{j}$, and the numerator $N$ is an arbitrary polynomial in these momenta. 
Step 1: The integral is written in Feynman-parameter representation and split into $n+1$ primary sectors as described in Ref. [29]. After eliminating the usual $\delta$ function $\delta\left(1-\sum_{i=1}^{n+1} x_{i}\right)$, each primary sector gives rise to a Feynman-parameter integral over the unit cube in $n$ dimensions of the form

$$
\int_{[0,1]^{n}} \mathrm{~d}^{n} \vec{x} \frac{f(\vec{x})}{[D(\vec{x})]^{e}}, \quad D(\vec{x})=s P_{s}(\vec{x})+r P_{r}(\vec{x})+\ldots+m^{2} P_{m}(\vec{x})+\lambda^{2} P_{\lambda}(\vec{x}),
$$

where the resulting denominator $D(\vec{x})$ is split into polynomials according to the hierarchy of scales in the diagram, which is assumed to be $s \gg r \gg \ldots \gg m^{2} \gg \lambda^{2}$ in (3.5). Note that in order to extract the angular-dependent logarithms $\log (s / r)$ with $r=t, u, \ldots$, we compute the integrals in the Euclidean region in various limits of the type $s \gg t=u$, $s=t \gg u$, etc., where we separate the energy scales in various ways.

Step 2: The polynomials in (3.5) have various zeros if subsets of Feynman parameters vanish, e.g.

$$
P(\vec{x})=0, \quad \text { at } \quad x_{1}=\ldots=x_{q}=0,
$$

which give rise to mass singularities. In the presence of such a singularity, the polynomial can be written as

$$
P(\vec{x})=\sum_{i=1}^{q} x_{i} P_{i}(\vec{x})
$$

In order to separate the singularity associated to (3.6) from other overlapping singularities, we decompose the corresponding integration domain $[0,1]^{q}$ into $q$ subsectors $\Omega_{j}$ with $x_{j}>x_{i \neq j}$, and in each subsector $\Omega_{j}$ we perform variable transformations $x_{i} \rightarrow x_{j} x_{i}^{\prime}$ for all $i \neq j$, which remap $\Omega_{j} \rightarrow[0,1]^{q}$ and bring the polynomial (3.7) into the form

$$
P(\vec{x})=\left[P_{j}(\vec{x})+\sum_{\substack{i=1 \\ i \neq j}}^{q} x_{i}^{\prime} P_{i}(\vec{x})\right] x_{j}
$$

where the variable $x_{j}$ is factorized.

Step 3: Recursive application of step 2 permits to factorize all zeros at all scales, until the denominator assumes the form ${ }^{5}$

$$
\hat{D}(\vec{x})=\left\{\ldots\left[s \hat{P}_{s}(\vec{x}) \prod_{j=1}^{n} x_{j}^{a_{j}}+r \hat{P}_{r}(\vec{x})\right] \prod_{k=1}^{n} x_{k}^{b_{k}}+\ldots+m^{2} \hat{P}_{m}(\vec{x})\right\} \prod_{l=1}^{n} x_{l}^{c_{l}}+\lambda^{2} \hat{P}_{\lambda}(\vec{x}),
$$

where $a_{k}, b_{k}, c_{k}$ are positive integers. In (3.9) all Feynman parameters that give rise to mass singularities are factorized and the polynomials $\hat{P}$ are non-vanishing. This allows for a simple power counting of the logarithmically divergent integrations.

Step 4: All logarithms of ratios of scales can now be extracted in $\mathrm{NLL}_{\mathrm{a}}$ approximation by analytical integration, where the polynomials $\hat{P}$ can be treated as constants $\hat{P}(\vec{x}) \simeq$ $\hat{P}(\overrightarrow{0})$. At present, explicit results are available [33] for the special class of integrals where

\footnotetext{
${ }^{5}$ In general the denominator assumes the form $D(\vec{x})=\hat{D}(\vec{x}) \prod_{i=1}^{n} x_{i}^{d_{i}}$, and the overall factorized Feynman parameters $x_{i}$ with $d_{i}>0$ can be cancelled by corresponding terms in the numerator.
} 
the various subsets of parameters $\left\{x_{j} \mid a_{j}>0\right\},\left\{x_{k} \mid b_{k}>0\right\}$, etc. that are associated to different mass scales are disjoint. As an example, for the case of a hierarchy of four different scales $s \gg r \gg M^{2} \gg \lambda^{2}$, we have integrals of the type ${ }^{6}$

$$
\begin{aligned}
& \int_{0}^{1} \mathrm{~d}^{l} \vec{x} \int_{0}^{1} \mathrm{~d}^{m} \vec{y} \int_{0}^{1} \mathrm{~d}^{n} \vec{z} \frac{s^{e}\left[\prod_{i=1}^{l} x_{i} \prod_{j=1}^{m} y_{j} \prod_{k=1}^{n} z_{k}\right]^{e-1}}{\left.\left\{\left(s \prod_{i=1}^{l} x_{i}+r\right) \prod_{j=1}^{m} y_{j}+M^{2}\right] \prod_{k=1}^{n} z_{k}+\lambda^{2}\right\}^{e}} \\
& \stackrel{\mathrm{NLL}_{\mathrm{a}}}{\sum_{p=0}^{n}} \sum_{q=0}^{n+m-p} \frac{1}{p !} \frac{1}{q !} \frac{1}{(N-p-q) !} \log ^{p}\left(\frac{M^{2}}{\lambda^{2}}\right) \log ^{q}\left(\frac{r}{M^{2}}\right) \log ^{N-p-q}\left(\frac{s}{r}\right),
\end{aligned}
$$

where $N=l+m+n$ and $e \geq 1$. Such integrals permitted us to calculate all diagrams listed in App. B.2 except for the ladder diagrams with simultaneous photon-mass and externalmass singularities, since these diagrams lead to integrals where the various subsets of parameters $\left\{x_{j} \mid a_{j}>0\right\},\left\{x_{k} \mid b_{k}>0\right\}$, etc. are not disjoint. Such integrals have not been solved so far, since it was more convenient to perform the calculation using the Sudakov method.

\section{Results}

In this section we present results for one- and two-loop Feynman diagrams evaluated in the high-energy limit (2.3) using the eikonal approximation (eik) and to next-to-leading logarithmic angular-dependent (NLL ${ }_{\mathrm{a}}$ ) accuracy (2.4). All results are split according to (2.13) into contributions corresponding to $\lambda=M$ and remaining $\Delta$ contributions, which originate from the gap $\lambda \ll M$ in the gauge sector.

In Sect. 4.1] we first recall the one-loop results [16], we then present in Sect. 4.2] our results for various subsets of two-loop diagrams and for the complete two-loop corrections, and in Sect. 4.3 we discuss the exponentiation of these logarithmic corrections. Explicit results for the individual one- and two-loop integrals can be found in App. B

\subsection{One-loop results}

Within the 't Hooft-Feynman gauge, the one-loop LL and angular-dependent NLL [ [16] originate from diagrams where a gauge boson $V^{a}=\gamma, \mathrm{Z}, \mathrm{W}^{ \pm}$is exchanged between two different on-shell external legs $j \neq k$,

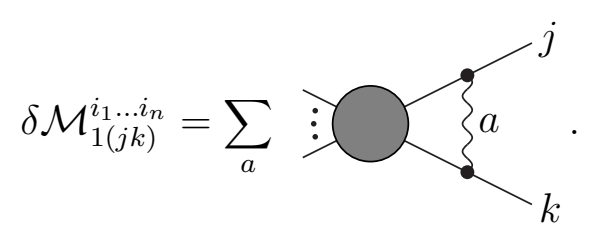

In the eikonal approximation, these yield

$$
\delta \mathcal{M}_{1(j k)}^{i_{1} \ldots i_{n}} \stackrel{\text { eik }}{=} \frac{\alpha}{4 \pi} \mathcal{M}_{0}^{i_{1} \ldots i_{j}^{\prime} \ldots i_{k}^{\prime} \ldots i_{n}} \sum_{a} I_{i_{j}^{\prime} i_{j}}^{a} I_{i_{k}^{\prime} i_{k}}^{\bar{a}} S\left(M_{a} ; p_{j}, p_{k}\right),
$$

\footnotetext{
${ }^{6}$ Here we consider all terms with the total power of logarithms equal to $N=l+m+n$, but in NLL $_{\text {a }}$ approximation we only need the contributions with $N-p-q \leq 1$.
} 
where the integral $S\left(M_{a} ; p_{j}, p_{k}\right)$ defined in (B.2) can be decomposed into

$$
S\left(M_{a} ; p_{j}, p_{k}\right) \stackrel{\mathrm{NLL}}{=} E\left(M_{a} ; m_{j}\right)+E\left(M_{a} ; m_{k}\right)+R\left(M_{a} ; p_{j}, p_{k}\right),
$$

with

$$
\begin{aligned}
E\left(\lambda ; m_{j}\right) & =\frac{1}{2} \log ^{2} \frac{\lambda^{2}}{s}-\frac{1}{2} \log ^{2} \frac{\lambda^{2}}{m_{j}^{2}}, \quad E\left(M ; m_{j}\right)=E(M)=\frac{1}{2} \log ^{2} \frac{M^{2}}{s}, \\
R\left(M_{a} ; p_{j}, p_{k}\right) & =2 \log \frac{M_{a}^{2}}{s} \log \frac{s}{\left|2 p_{j} p_{k}\right|} .
\end{aligned}
$$

The functions $E$ depend only on the energy scale $s$, on the internal masses $M_{a}$, and on the masses $m_{j, k}$ of the external lines, and do not give rise to correlations between the two external legs $j, k$ in the sum (4.5), whereas the function $R$ contains logarithms of $p_{j} p_{k} / s$ depending on the angle between the momenta $p_{j}$ and $p_{k}$, but is independent of the external masses.

The complete one-loop correction is obtained by taking the sum over all pairs of external legs, and the part originating from the functions $E$ can be simplified by using the charge-conservation identity (2.6). This yields

$$
\begin{aligned}
\delta \mathcal{M}_{1}^{i_{1} \ldots i_{n}}= & \frac{1}{2} \sum_{\substack{j, k=1 \\
k \neq j}}^{n} \delta \mathcal{M}_{1(j k)}^{i_{1} \ldots i_{n}} \\
= & -\frac{\alpha}{4 \pi}\left\{\sum_{j=1}^{n} \mathcal{M}_{0}^{i_{1} \ldots i_{j}^{\prime} \ldots i_{n}}\left[C_{i_{j}^{\prime} i_{j}}^{\mathrm{ew}} E(M)+\delta_{i_{j}^{\prime} i_{j}} Q_{i_{j}}^{2} \Delta E\left(\lambda ; m_{j}\right)\right]\right. \\
& -\frac{1}{2} \sum_{\substack{j, k=1 \\
k \neq j}}^{n} \mathcal{M}_{0}^{i_{1} \ldots i_{j}^{\prime} \ldots i_{k}^{\prime} \ldots i_{n}}\left[\sum_{a=\gamma, Z, W} I_{i_{j}^{\prime} i_{j} i_{j}}^{a} I_{i_{k}^{\prime} i_{k}}^{\bar{a}} R\left(M ; p_{j}, p_{k}\right)\right. \\
& \left.\left.\quad+\delta_{i_{j}^{\prime} i_{j}} Q_{i_{j}} \delta_{i_{k}^{\prime} i_{k}} Q_{i_{k}} \Delta R\left(\lambda ; p_{j}, p_{k}\right)\right]\right\},
\end{aligned}
$$

where $C^{\mathrm{ew}}$ represents the electroweak Casimir operator defined in (A.5), $Q_{i_{j}}$ is the eigenvalue of the charge operator $I_{i_{j}^{\prime} i_{j}}^{\gamma}=-Q_{i_{j}} \delta_{i_{j}^{\prime} i_{j}}$, and

$$
\begin{gathered}
\Delta E\left(\lambda ; m_{j}\right)=E\left(\lambda ; m_{j}\right)-E\left(M ; m_{j}\right)=\log \left(\frac{m_{j}^{2}}{s}\right) \log \left(\frac{\lambda^{2}}{M^{2}}\right)-\frac{1}{2} \log ^{2}\left(\frac{m_{j}^{2}}{M^{2}}\right), \\
\Delta R\left(\lambda ; p_{j}, p_{k}\right)=R\left(\lambda ; p_{j}, p_{k}\right)-R\left(M ; p_{j}, p_{k}\right)=2 \log \left(\frac{\lambda^{2}}{M^{2}}\right) \log \left(\frac{s}{\left|2 p_{j} p_{k}\right|}\right) .
\end{gathered}
$$

In order to discuss the two-loop results in the next section, it is useful to rewrite the one-loop result (4.5) in matrix form. To this end we introduce the following notation

$$
\begin{aligned}
& \mathcal{M} \equiv \mathcal{M}^{i_{1} \ldots i_{n}}, \quad \mathcal{M} I^{a}(k) \equiv \mathcal{M}^{i_{1} \ldots i_{k}^{\prime} \ldots i_{n}} I_{i_{k}^{\prime} i_{k}}^{a}, \\
& \mathcal{M} I^{a}(k) I^{b}(k) \equiv \mathcal{M}^{i_{1} \ldots i_{k}^{\prime \prime} \ldots i_{n}} I_{i_{k}^{\prime \prime} i_{k}^{\prime}}^{a} I_{i_{k}^{\prime} i_{k}}^{b}, \quad \text { etc. }
\end{aligned}
$$

where the generators $I^{a}(k)$ are matrices acting on the indices corresponding to the external leg $k$ of the matrix element, with commutation relations

$$
\left[I^{a}(k), I^{b}(l)\right]=\delta_{k l} \sum_{c=\gamma, Z, W^{ \pm}} I^{c}(k) I_{c b}^{a} .
$$


Using this notation also for other matrices as $C^{\mathrm{ew}}(k)$ or $Q(k)$, the result (4.5) can be rewritten as

$$
\delta \mathcal{M}_{1}=\mathcal{M}_{0} \delta_{\mathrm{EW}}=\mathcal{M}_{0}\left(\delta_{\text {sew }}+\delta_{\text {sem }}\right)
$$

where the complete electroweak (EW) result is split into a symmetric electroweak (sew) part

$$
\begin{aligned}
\delta_{\mathrm{sew}} & =\left.\delta_{\mathrm{EW}}\right|_{\lambda=M} \\
& =\frac{\alpha}{4 \pi}\left\{-\frac{1}{2} \sum_{j=1}^{n} C^{\mathrm{ew}}(j) \log ^{2} \frac{M^{2}}{s}+\sum_{\substack{j, k=1 \\
k \neq j}}^{n} \sum_{\substack{a=\gamma, \mathrm{Z}, \mathrm{W}^{ \pm} \\
I^{a}}}(j) I^{\bar{a}}(k) \log \frac{s}{\left|2 p_{j} p_{k}\right|} \log \frac{M^{2}}{s}\right\},
\end{aligned}
$$

which corresponds to the case $\lambda=M$ and is manifestly $\mathrm{SU}(2) \times \mathrm{U}(1)$ symmetric, and a remaining subtracted electromagnetic (sem) part

$$
\begin{aligned}
\delta_{\mathrm{sem}}= & \Delta \delta_{\mathrm{EW}}=\delta_{\mathrm{EW}}-\left.\delta_{\mathrm{EW}}\right|_{\lambda=M}=\frac{\alpha}{4 \pi}\left\{-\frac{1}{2} \sum_{j=1}^{n} Q^{2}(j)\left[2 \log \frac{m_{j}^{2}}{s} \log \frac{\lambda^{2}}{M^{2}}-\log ^{2} \frac{m_{j}^{2}}{M^{2}}\right]\right. \\
& \left.+\sum_{\substack{j, k=1 \\
k \neq j}}^{n} Q(j) Q(k) \log \frac{s}{\left|2 p_{j} p_{k}\right|} \log \frac{\lambda^{2}}{M^{2}}\right\},
\end{aligned}
$$

which originates from the gap $\lambda \ll M$, i.e. from the $\Delta$ terms in (4.5), and is $\mathrm{U}(1)_{\mathrm{em}}$ symmetric.

\subsection{Two-loop results}

In the following we present results for the two-loop diagrams (2.7) combined into three subsets where the soft-collinear gauge bosons couple to two, three, or four of the $n$ on-shell external lines, respectively.

The two-loop results are decomposed into reducible contributions, which involve products of the one-loop integrals (4.3), plus remaining irreducible parts. This decomposition is based on the explicit two-loop results in the $\mathrm{NLL}_{\mathrm{a}}$ approximation (2.4) given in App. B.2 and the relations given in App. C.

\section{Terms from two external lines}

We begin by considering the contributions

$$
\delta \mathcal{M}_{2(j k)}^{i_{1} \ldots i_{n}}=\sum_{a, b}\left\{D_{2 \mathrm{~L}, j k}^{a b}+D_{2 \mathrm{C}, j k}^{a b}+\sum_{c}\left[D_{2 \mathrm{Y}, j k}^{a b c}+D_{2 \mathrm{Y}, k j}^{a b c}\right]\right\},
$$

corresponding to the diagrams

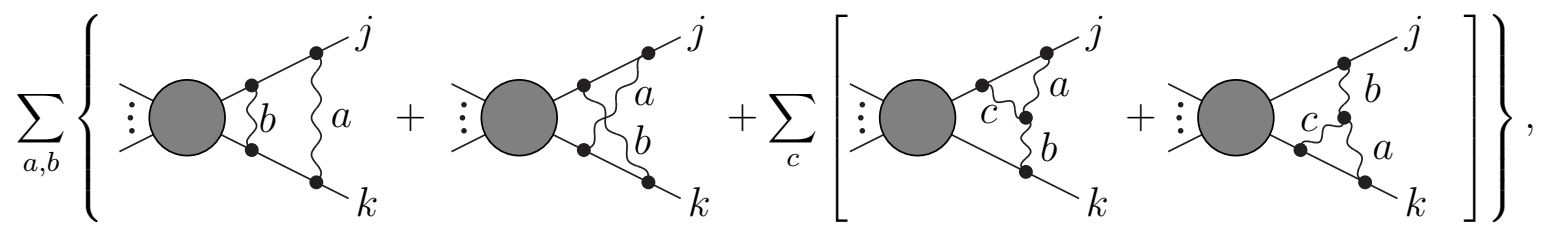


i.e. diagrams with $n$ on-shell external legs and soft-collinear gauge bosons $V^{a}, V^{b}, V^{c}=$ $\gamma, \mathrm{Z}, \mathrm{W}$ exchanged between only two of these external lines. These yield

$$
\begin{aligned}
\delta \mathcal{M}_{2(j k)}^{i_{1} \ldots i_{n}} & =\left(\frac{\alpha}{4 \pi}\right)^{2} \mathcal{M}_{0}^{i_{1} \ldots i_{j}^{\prime} \ldots i_{k}^{\prime} \ldots i_{n}} \sum_{a, b}\left\{\left(I^{\bar{b}} I^{\bar{a}}\right)_{i_{j}^{\prime} i_{j}}\left(I^{b} I^{a}\right)_{i_{k}^{\prime} i_{k}} S_{2 \mathrm{~L}}\left(M_{a}, M_{b} ; p_{j}, p_{k}\right)\right. \\
& +\left(I^{\bar{b}} I^{\bar{a}}\right)_{i_{j}^{\prime} i_{j}}\left(I^{a} I^{b}\right)_{i_{k}^{\prime} i_{k}} S_{2 \mathrm{C}}\left(M_{a}, M_{b} ; p_{j}, p_{k}\right) \\
& \left.+\sum_{c}\left[\left(I^{\bar{c}} I^{\bar{a}}\right)_{i_{j}^{\prime} i_{j}} I_{i_{k}^{\prime} i_{k}}^{b} I_{b c}^{a} S_{2 \mathrm{Y}}\left(M_{a}, M_{b}, M_{c} ; p_{j}, p_{k}\right)+(j \leftrightarrow k)\right]\right\} \\
\mathrm{NLLLa}_{\underline{a}} & \left(\frac{\alpha}{4 \pi}\right)^{2} \mathcal{M}_{0}^{i_{1} \ldots i_{j}^{\prime} \ldots i_{k}^{\prime} \ldots i_{n}}\left\{\frac{1}{2} \sum_{a, b}\left(I^{\bar{b}} I^{\bar{a}}\right)_{i_{j}^{\prime} i_{j}}\left(I^{b} I^{a}\right)_{i_{k}^{\prime} i_{k}}\left[S\left(M ; p_{j}, p_{k}\right)\right]^{2}\right. \\
& +\sum_{a}\left(I^{\bar{a}} I^{\gamma}\right)_{i_{j}^{\prime} i_{j}}\left(I^{a} I^{\gamma}\right)_{i_{k}^{\prime} i_{k}} S\left(M ; p_{j}, p_{k}\right) \Delta S\left(\lambda ; p_{j}, p_{k}\right) \\
& +\frac{1}{2}\left(I^{\gamma} I^{\gamma}\right)_{i_{j}^{\prime} i_{j}}\left(I^{\gamma} I^{\gamma}\right)_{i_{k}^{\prime} i_{k}}\left[\Delta S\left(\lambda ; p_{j}, p_{k}\right)\right]^{2} \\
& \left.+\frac{1}{2} \sum_{a, c} \sum_{h=1}^{n} I_{i_{h}^{\prime} i_{h}}^{\bar{a}}\left[I_{i_{j}^{\prime} i_{j}}^{\bar{c}} I_{i_{k}^{\prime} i_{k}}^{\gamma} I_{\gamma a}^{c} \Delta S_{2 \mathrm{C}}\left(M, \lambda ; p_{j}, p_{k}\right)+(j \leftrightarrow k)\right]\right\}
\end{aligned}
$$

where we have made use of the identities (2.6), (2.13), (A.3), (C.1), (C.2), and (C.3).

\section{Terms from three external lines}

Here we consider the contributions

$$
\delta \mathcal{M}_{2(j k l)}^{i_{1} \ldots i_{n}}=\sum_{a, b}\left\{\sum_{\pi(j, k, l)} D_{3 \mathrm{~L}, j k l}^{a b}+\sum_{c} D_{3 \mathrm{Y}, j k l}^{a b c}\right\},
$$

where we sum over all six permutations $\pi(j, k, l)$ of external lines $j, k, l$. These contributions correspond to the diagrams

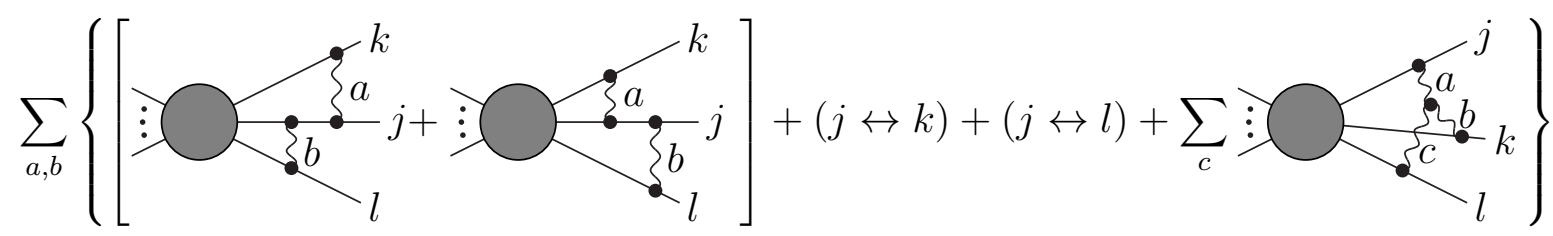

with exchange of soft-collinear gauge bosons $V^{a}, V^{b}, V^{c}=\gamma, \mathrm{Z}, \mathrm{W}$ between three external on-shell lines, which yield

$$
\begin{aligned}
\delta \mathcal{M}_{2(j k l)}^{i_{1} \ldots i_{n}} \stackrel{\text { eik }}{=} & \left(\frac{\alpha}{4 \pi}\right)^{2} \mathcal{M}_{0}^{i_{1} \ldots i_{j}^{\prime} \ldots i_{k}^{\prime} \ldots i_{l}^{\prime} \ldots i_{n}} \\
& \times\left\{\sum_{\pi(j, k, l)} \sum_{a, b}\left(I^{b} I^{a}\right)_{i_{j}^{\prime} i_{j}} I_{i_{k}^{\prime} i_{k}}^{\bar{a}} I_{i_{l}^{\prime} i_{l}}^{\bar{b}} S_{3 \mathrm{~L}}\left(M_{a}, M_{b} ; p_{j}, p_{k}, p_{l}\right)\right. \\
& \left.+\sum_{a, b, c} I_{i_{j}^{\prime} i_{j}}^{\bar{a}} I_{b c}^{a} I_{i_{k}^{\prime} i_{k} i_{k}}^{b} I_{i_{l}^{\prime} i_{l}}^{\bar{c}} S_{3 \mathrm{Y}}\left(M_{a}, M_{b}, M_{c} ; p_{j}, p_{k}, p_{l}\right)\right\}
\end{aligned}
$$




$$
\begin{aligned}
\stackrel{\mathrm{NLL}_{a}}{=} & \left(\frac{\alpha}{4 \pi}\right)^{2} \mathcal{M}_{0}^{i_{1} \ldots i_{j}^{\prime} \ldots i_{k}^{\prime} \ldots i_{l}^{\prime} \ldots i_{n}} \\
& \times \sum_{\pi(j, k, l)}\left\{\frac{1}{2} \sum_{a, b}\left(I^{b} I^{a}\right)_{i_{j}^{\prime} i_{j}} I_{i_{k}^{\prime} i_{k}}^{\bar{a}} I_{i_{l}^{\prime} i_{l}}^{\bar{b}} S\left(M ; p_{j}, p_{k}\right) S\left(M ; p_{j}, p_{l}\right)\right. \\
& +\sum_{a}\left(I^{a} I^{\gamma}\right)_{i_{j}^{\prime} i_{j}} I_{i_{k}^{\prime} i_{k}}^{\bar{a}} I_{i_{l}^{\prime} i_{l}}^{\gamma} S\left(M ; p_{j}, p_{k}\right) \Delta S\left(\lambda ; p_{j}, p_{l}\right) \\
& +\frac{1}{2}\left(I^{\gamma} I^{\gamma}\right)_{i_{j}^{\prime} i_{j}} I_{i_{k}^{\prime} i_{k}}^{\gamma} I_{i_{l}^{\prime} i_{l}}^{\gamma} \Delta S\left(\lambda ; p_{j}, p_{k}\right) \Delta S\left(\lambda ; p_{j}, p_{l}\right) \\
& \left.-\frac{1}{2} \sum_{a, c} I_{i_{j}^{\prime} i_{j}}^{\bar{c}} I_{\gamma a}^{c} I_{i_{k}^{\prime} i_{k}}^{\bar{a}} I_{i_{l}^{\prime} i_{l}}^{\gamma} \Delta S_{3 \mathrm{~L}}\left(M, \lambda ; p_{j}, p_{k}, p_{l}\right)\right\},
\end{aligned}
$$

where we used (2.13), (A.3), (C.4), (C.5.6), and (C.7).

\section{Terms from four external lines}

Finally, we have the contributions

$$
\delta \mathcal{M}_{2(j k l m)}^{i_{1} \ldots i_{n}}=\sum_{a, b} D_{4 \mathrm{~L}, j k l m}^{a b}=\sum_{a, b} \vdots \sum_{\{b}^{j} b_{m}^{j},
$$

originating from gauge bosons coupling to four external legs, which reduce according to (B.32) to simple products of one-loop integrals

$$
\delta \mathcal{M}_{2(j k l m)}^{i_{1} \ldots i_{n}} \stackrel{\text { eik }}{=}\left(\frac{\alpha}{4 \pi}\right)^{2} \mathcal{M}_{0}^{i_{1} \ldots i_{j}^{\prime} \ldots i_{k}^{\prime} \ldots i_{l}^{\prime} \ldots i_{m}^{\prime} \ldots i_{n}} \sum_{a, b} I_{i_{j}^{\prime} i_{j}}^{a} I_{i_{k}^{\prime} i_{k}}^{\bar{a}} I_{i_{l}^{\prime} i_{l}}^{b} I_{i_{m}^{\prime} i_{m}}^{\bar{b}} S\left(M_{a} ; p_{j}, p_{k}\right) S\left(M_{b} ; p_{l}, p_{m}\right) .
$$

\section{Complete two-loop correction}

We now combine the contributions from the above subsets of diagrams into the complete virtual two-loop correction to an arbitrary process involving $n$ on-shell external legs as follows

$$
\delta \mathcal{M}_{2}^{i_{1} \ldots i_{n}}=\frac{1}{2} \sum_{j, k}^{n} \mathcal{M}_{2(j k)}^{i_{1} \ldots i_{n}}+\frac{1}{6} \sum_{j, k, l}^{n} \mathcal{M}_{2(j k l)}^{i_{1} \ldots i_{n}}+\frac{1}{8} \sum_{j, k, l, m}^{n} \mathcal{M}_{2(j k l m)}^{i_{1} \ldots i_{n}},
$$

where we have to sum over all combinations of external legs with appropriate symmetry factors. The primes indicate that the sums include only terms with different external legs, i.e.

$$
\sum_{j, k}^{n}:=\sum_{\substack{j, k=1 \\ k \neq j}}^{n}, \quad \sum_{j, k, l}^{n}:=\sum_{\substack{j, k, l=1 \\ k \neq j ; l \neq j, k}}^{n}, \quad \sum_{j, k, l, m}^{n}:=\sum_{\substack{j, k, l, m=1 \\ k \neq j ; l \neq j, k ; m \neq l, j, k}}^{n} .
$$

We first consider the irreducible contributions to (4.20), i.e. the contributions from $\Delta S_{2 \mathrm{C}}$ in (4.14) and $\Delta S_{3 \mathrm{~L}}$ in (4.17), which could not be expressed as products of one-loop integrals. These contributions cancel,

$$
\delta \mathcal{M}_{2, \text { irr. }}^{i_{1} \ldots i_{n}}=\left(\frac{\alpha}{4 \pi}\right)^{2} \frac{1}{2} \sum_{j, k, l}^{n} \mathcal{M}_{0}^{i_{1} \ldots i_{j}^{\prime} \ldots i_{k}^{\prime} \ldots i_{l}^{\prime} \ldots i_{n}} \sum_{a, c} I_{\gamma a}^{c} I_{i_{j}^{\prime} i_{j}}^{\bar{c}} I_{i_{k}^{\prime} i_{k}}^{\gamma} I_{i_{l}^{\prime} i_{l}}^{\bar{a}}
$$




$$
\times\left[\Delta S_{2 \mathrm{C}}\left(M, \lambda ; p_{j}, p_{k}\right)-\Delta S_{3 \mathrm{~L}}\left(M, \lambda ; p_{j}, p_{l}, p_{k}\right)\right]_{\stackrel{\mathrm{NLL}}{=}}^{=} 0
$$

because of (C.8). The complete two-loop correction is thus given by the reducible contributions to (4.14), (4.17), and (4.19), i.e. contributions from products of one-loop integrals $S$. These yield

$$
\begin{aligned}
& \delta \mathcal{M}_{2}^{i_{1} \ldots i_{n}} \stackrel{\mathrm{NLL}_{\mathrm{a}}}{=}\left(\frac{\alpha}{4 \pi}\right)^{2}\left\{\frac{1}{8} \sum_{j, k, l, m}^{n} \mathcal{M}_{0}^{i_{1} \ldots i_{j}^{\prime} \ldots i_{k}^{\prime} \ldots i_{l}^{\prime} \ldots i_{m}^{\prime} \ldots i_{n}} \sum_{a, b} I_{i_{j}^{\prime} i_{j}}^{a} I_{i_{k}^{\prime} i_{k}}^{\bar{a}} I_{i_{l}^{\prime} i_{l}}^{b} I_{i_{m}^{\prime} i_{m}}^{\bar{b}}\right. \\
& \times S\left(M_{a} ; p_{j}, p_{k}\right) S\left(M_{b} ; p_{l}, p_{m}\right) \\
& +\sum_{j, k, l}^{n} \mathcal{M}_{0}^{i_{1} \ldots i_{j}^{\prime} \ldots i_{k}^{\prime} \ldots i_{l}^{\prime} \ldots i_{n}}\left[\frac{1}{2} \sum_{a, b}\left(I^{b} I^{a}\right)_{i_{j}^{\prime} i_{j}} I_{i_{k}^{\prime} i_{k}}^{\bar{a}} I_{i_{l}^{\prime} i_{l}}^{\bar{b}} S\left(M ; p_{j}, p_{k}\right) S\left(M ; p_{j}, p_{l}\right)\right. \\
& +\sum_{a}\left(I^{a} I^{\gamma}\right)_{i_{j}^{\prime} i_{j}} I_{i_{k}^{\prime} i_{k}}^{\bar{a}} I_{i_{l}^{\prime} i_{l}}^{\gamma} S\left(M ; p_{j}, p_{k}\right) \Delta S\left(\lambda ; p_{j}, p_{l}\right) \\
& \left.+\frac{1}{2}\left(I^{\gamma} I^{\gamma}\right)_{i_{j}^{\prime} i_{j}} I_{i_{k}^{\prime} i_{k}}^{\gamma} I_{i_{l}^{\prime} i_{l}}^{\gamma} \Delta S\left(\lambda ; p_{j}, p_{k}\right) \Delta S\left(\lambda ; p_{j}, p_{l}\right)\right] \\
& +\sum_{j, k}^{n} \mathcal{M}_{0}^{i_{1} \ldots i_{j}^{\prime} \ldots i_{k}^{\prime} \ldots i_{n}}\left[\frac{1}{4} \sum_{a, b}\left(I^{\bar{b}} I^{\bar{a}}\right)_{i_{j}^{\prime} i_{j}}\left(I^{b} I^{a}\right)_{i_{k}^{\prime} i_{k}}\left[S\left(M ; p_{j}, p_{k}\right)\right]^{2}\right. \\
& +\frac{1}{2} \sum_{a}\left(I^{\bar{a}} I^{\gamma}\right)_{i_{j}^{\prime} i_{j}}\left(I^{a} I^{\gamma}\right)_{i_{k}^{\prime} i_{k}} S\left(M ; p_{j}, p_{k}\right) \Delta S\left(\lambda ; p_{j}, p_{k}\right) \\
& \left.\left.+\frac{1}{4}\left(I^{\gamma} I^{\gamma}\right)_{i_{j}^{\prime} i_{j}}\left(I^{\gamma} I^{\gamma}\right)_{i_{k}^{\prime} i_{k}}\left[\Delta S\left(\lambda ; p_{j}, p_{k}\right)\right]^{2}\right]\right\} \\
& \stackrel{\mathrm{NLL}_{\mathrm{a}}}{=} \frac{1}{2}\left(\frac{\alpha}{4 \pi}\right)^{2} \sum_{j, k}^{n} \mathcal{M}_{0}^{i_{1} \ldots i_{j}^{\prime} \ldots i_{k}^{\prime} \ldots i_{n}}\left[\sum_{a}\left(I^{a} I^{\bar{a}}\right)_{i_{j}^{\prime} i_{j}} E(M)+\left(I^{\gamma} I^{\gamma}\right)_{i_{j}^{\prime} i_{j}} \Delta E\left(\lambda ; m_{j}\right)\right] \\
& \times\left[\sum_{b}\left(I^{b} I^{\bar{b}}\right)_{i_{k}^{\prime} i_{k}} E(M)+\left(I^{\gamma} I^{\gamma}\right)_{i_{k}^{\prime} i_{k}} \Delta E\left(\lambda ; m_{k}\right)\right] \\
& +\frac{1}{2}\left(\frac{\alpha}{4 \pi}\right)^{2} \sum_{j=1}^{n} \mathcal{M}_{0}^{i_{1} \ldots i_{j}^{\prime} \ldots i_{n}}\left[\sum_{a, b}\left(I^{a} I^{\bar{a}} I^{b} I^{\bar{b}}\right)_{i_{j}^{\prime} i_{j}}[E(M)]^{2}\right. \\
& \left.+2 \sum_{a}\left(I^{a} I^{\bar{a}} I^{\gamma} I^{\gamma}\right)_{i_{j}^{\prime} i_{j}} E(M) \Delta E\left(\lambda ; m_{j}\right)+\left(I^{\gamma} I^{\gamma} I^{\gamma} I^{\gamma}\right)_{i_{j}^{\prime} i_{j}}\left[\Delta E\left(\lambda ; m_{j}\right)\right]^{2}\right] \\
& -\frac{1}{2}\left(\frac{\alpha}{4 \pi}\right)^{2} \sum_{j, k, l}^{n} \mathcal{M}_{0}^{i_{1} \ldots i_{j}^{\prime} \ldots i_{k}^{\prime} \ldots i_{l}^{\prime} \ldots i_{n}}\left[\sum_{b}\left(I^{b} I^{\bar{b}}\right)_{i_{l}^{\prime} i_{l}} E(M)+\left(I^{\gamma} I^{\gamma}\right)_{i_{l}^{\prime} i_{l}} \Delta E\left(\lambda ; m_{l}\right)\right] \\
& \times\left[\sum_{a} I_{i_{j}^{\prime} i_{j}}^{a} I_{i_{k}^{\prime} i_{k}}^{\bar{a}} R\left(M ; p_{j}, p_{k}\right)+I_{i_{j}^{\prime} i_{j}}^{\gamma} I_{i_{k}^{\prime} i_{k}}^{\gamma} \Delta R\left(\lambda ; p_{j}, p_{k}\right)\right] \\
& -\left(\frac{\alpha}{4 \pi}\right)^{2} \sum_{j, k}^{n} \mathcal{M}_{0}^{i_{1} \ldots i_{j}^{\prime} \ldots i_{k}^{\prime} \ldots i_{n}}\left[\sum_{a, b}\left(I^{a} I^{b} I^{\bar{b}}\right)_{i_{j}^{\prime} i_{j}} I_{i_{k}^{\prime} i_{k}}^{\bar{a}} E(M) R\left(M ; p_{j}, p_{k}\right)\right. \\
& +\sum_{a}\left(I^{b} I^{\bar{b}} I^{\gamma}\right)_{i_{j}^{\prime} i_{j}} I_{i_{k}^{\prime} i_{k}}^{\gamma} E(M) \Delta R\left(\lambda ; p_{j}, p_{k}\right) \\
& +\sum_{a}\left(I^{a} I^{\gamma} I^{\gamma}\right)_{i_{j}^{\prime} i_{j}} I_{i_{k}^{\prime} i_{k}}^{\bar{a}} \Delta E\left(\lambda ; m_{j}\right) R\left(M ; p_{j}, p_{k}\right) \\
& \left.+\left(I^{\gamma} I^{\gamma} I^{\gamma}\right)_{i_{j}^{\prime} i_{j} i_{i^{\prime}} i_{k}}^{\gamma} \Delta E\left(\lambda ; m_{j}\right) \Delta R\left(\lambda ; p_{j}, p_{k}\right)\right],
\end{aligned}
$$


where we have used (2.6), (4.3), (A.3), (A.4), and the fact that $R\left(M_{a} ; p_{j}, p_{k}\right)$ is symmetric with respect to interchanging $j \leftrightarrow k$. Note that the photonic coupling matrices $I^{\gamma} I^{\gamma}$ are to the right of $I^{a}$ in the term containing $\Delta E\left(\lambda ; m_{j}\right) R\left(M ; p_{j}, p_{k}\right)$.

Using $I_{i_{j}^{\prime} i_{j}}^{\gamma}=-Q_{i_{j}} \delta_{i_{j}^{\prime} i_{j}}$ and rewriting (4.23) in the compact operator form introduced in (4.7), results in

$$
\delta \mathcal{M}_{2} \stackrel{\mathrm{NLL}_{\mathrm{a}}}{=} \frac{1}{2} \mathcal{M}_{0}\left(\delta_{\text {sew }}^{2}+2 \delta_{\text {sew }} \delta_{\mathrm{sem}}+\delta_{\text {sem }}^{2}\right),
$$

where $\delta_{\text {sew }}$ and $\delta_{\text {sem }}$ are the operators defined in (4.10) and (4.11) and correspond to the symmetric electroweak and the subtracted electromagnetic parts of the one-loop corrections. It is important to note that these two operators have a non-vanishing commutator

$$
\left[\delta_{\text {sew }}, \delta_{\text {sem }}\right]=\mathcal{O}\left[\log \frac{\left|2 p_{k} p_{l}\right|}{s} \log ^{3-N} \frac{s}{M^{2}} \prod_{i=1}^{N} \log \frac{M^{2}}{m_{\text {light }, i}^{2}}\right], \quad N=1,2,
$$

where $m_{\text {light }}$ is either a light-fermion or a photon mass. This means that the ordering of the terms $\delta_{\text {sew }} \delta_{\text {sem }}$ in (4.24), which is determined by the contribution involving $I^{a} I^{\gamma} I^{\gamma}$ in (4.23), is relevant at the level of angular-dependent NLL. As we point out in the next section, the determination of this ordering constitutes an important aspect of our result and permits to discriminate between different exponentiation prescriptions for the electroweak corrections.

\subsection{Exponentiation}

If we combine the two-loop correction (4.24) with the one-loop correction (4.9) and the Born amplitude, to two-loop $\mathrm{NLL}_{\mathrm{a}}$ accuracy we find the exponentiated form

$$
\mathcal{M}_{2}=\mathcal{M}_{0}+\delta \mathcal{M}_{1}+\delta \mathcal{M}_{2} \stackrel{N_{L L a}}{=} \mathcal{M}_{0} \exp \left(\delta_{\text {sew }}\right) \exp \left(\delta_{\text {sem }}\right) .
$$

In particular, the form of the two-loop correction operator $\left(\delta_{\text {sew }}^{2}+2 \delta_{\text {sew }} \delta_{\text {sem }}+\delta_{\text {sem }}^{2}\right) / 2$ implies that the symmetric electroweak part $\delta_{\text {sew }}$ and the subtracted electromagnetic part $\delta_{\text {sem }}$ exponentiate separately, and that the latter exponential is external. This means that the charge operators in $\exp \left(\delta_{\mathrm{sem}}\right)$ can be identified with the charge eigenvalues of the external particles in the process.

At the level of the LL, this result confirms the exponentiation of the EWLC obtained with the IREE [20] and already checked for arbitrary processes by a two-loop calculation in the Coulomb gauge [ 26]. We found also agreement with the results of Refs. [24, 25] for the special case of the fermionic form factor corresponding to the decay $g \rightarrow f \bar{f}$ of an $\mathrm{SU}(2) \times \mathrm{U}(1)$ singlet $g$ into massless fermions. We have explicitly verified all results of Refs. [24, 25] by evaluating the subset of diagrams $D_{2 \mathrm{~L}}, D_{2 \mathrm{C}}$, and $D_{2 \mathrm{Y}}$ in (2.7) for the special case of massless external particles. At the level of angular-dependent NLL, our result is in agreement with the exponentiation prescriptions adopted in Refs. [19, 21] for massless fermionic processes, and extended in Ref. [23] to arbitrary processes.

This agreement indicates that, at least up to the level of angular-dependent NLL, a symmetric $\mathrm{SU}(2) \times \mathrm{U}(1)$ gauge theory matched with QED at the electroweak scale provides a correct physical picture for the resummation of EWLC in the high-energy limit. This picture has been formulated within the theoretical framework of the IREE 
[ 20, which describes the all-order leading-logarithmic dependence of matrix elements on the transverse-momentum cut-off $\mu_{\perp}$. This infrared scale $\mu_{\perp}$ is the crucial ingredient in order to avoid the difficulties related to the breaking of the $\mathrm{SU}(2) \times \mathrm{U}(1)$ symmetry that originate from the large mass gap $\lambda \ll M$ in the gauge-boson sector. In fact, the scale $\mu_{\perp}$ permits to separate two regimes of the electroweak theory both with exact gauge symmetry. The regime $\sqrt{s}>\mu_{\perp}>M$, which is insensitive to the gauge-boson masses and has $\mathrm{SU}(2) \times \mathrm{U}(1)$ symmetry, and the regime $M>\mu_{\perp}>\lambda$, where the weak gauge bosons are "frozen out" and only $\mathrm{U}(1)_{\mathrm{em}}$ symmetry is left.

To our knowledge, the IREE has been formulated only at the level of LL, and the application of the physical picture described above to the level of NLL relies on a weaker theoretical basis. At this level, the following two arguments can be used for the exponentiation of the next-to-leading logarithmic corrections. On the one hand, if $\lambda=M$ then the $\mathrm{SU}(2) \times \mathrm{U}(1)$ symmetry is restored in the gauge sector and one expects the exponentiation

$$
\lambda=M \Rightarrow \mathcal{M}_{2}=\mathcal{M}_{0} \exp \left[\delta_{\text {sew }}\right],
$$

as in a symmetric $\mathrm{SU}(2) \times \mathrm{U}(1)$ theory. This permits to predict the two-loop term proportional to $\delta_{\text {sew }}^{2}$ in (4.26) and implies that $\delta_{\text {sem }}=0$ at $\lambda=M$. On the other hand, the logarithms of the photon mass and light-fermion masses originate only from photons coupling to external legs and are expected to exponentiate as in QED. However, the QED results can be generalized to the electroweak corrections only if the contributions from virtual photons can be separated from those of the weak gauge bosons in a gauge-invariant way. This is the case only if $s=M^{2}$, where the logarithms of $s / M^{2}$ originating from virtual weak bosons vanish. Here one expects

$$
s=M^{2} \Rightarrow \mathcal{M}_{2}=\mathcal{M}_{0} \exp \left[\delta_{\mathrm{sem}}\right],
$$

where $\delta_{\text {sem }}$ corresponds to the QED corrections. This, together with (4.27), permits to determine $\delta_{\text {sem }}$ and the two-loop term proportional to $\delta_{\mathrm{sem}}^{2}$ in (4.26). However, we note that the above two conditions, (4.27) and (4.28), are not sufficient to determine the two-loop interference terms $\delta_{\text {sew }} \delta_{\text {sem }}$ between symmetric electroweak and subtracted electromagnetic contributions, which vanish in both cases $\lambda=M$ and $s=M^{2}$. These two-loop interference terms are an important result of our electroweak calculation for $s \gg M^{2} \gg \lambda^{2}$. In particular, they are crucial in order to predict the ordering of the two exponentials in (4.26), which starts to be non-trivial at the level of angular-dependent NLL as indicated by the commutator (4.25).

\section{Conclusions}

We have studied the two-loop asymptotic behaviour of virtual electroweak corrections to arbitrary processes involving light or heavy chiral fermions, transverse or longitudinal gauge bosons, or Higgs bosons. We have calculated the two-loop leading and angulardependent next-to-leading logarithmic contributions in a process-independent way in the region where all kinematic invariants are much larger than the electroweak scale. The relevant Feynman diagrams involving exchanges of soft and collinear virtual gauge bosons $\gamma, \mathrm{Z}$, and $\mathrm{W}^{ \pm}$between on-shell external legs have been evaluated in the eikonal approximation in the 't Hooft-Feynman gauge. 
Analytical expressions for the relevant two-loop integrals, which involve up two six different scales, have been calculated via two independent methods. On the one hand, we have evaluated the integrals in the Feynman-parameter representation using sector decomposition to isolate the mass singularities in the integrand and performing the integration in logarithmic approximation. This method was applied to all diagrams except for those ladder diagrams with simultaneous photon-mass and external-mass singularities. On the other hand, we have employed the well-known Sudakov method, which is very efficient for calculating diagrams with only one large energy scale but turns out to be more complicated for diagrams with more large energy scales. In particular, in the Sudakov approximation we did not succeed in calculating the diagram where three soft gauge bosons interacting via a Yang-Mills vertex couple to three different external legs. In all diagrams where both methods could be applied we found agreement at the angular-dependent next-to-leading logarithmic level.

In order to isolate the effects originating from the large mass-gap between the photon mass $\lambda$ and the weak-boson masses $M_{\mathrm{W}} \simeq M_{\mathrm{Z}} \simeq M$, which breaks the symmetry in the gauge-boson sector, the loop contributions depending on the photon mass have been split into a part corresponding to $\lambda=M$ and a remaining subtracted part. Combining the results from all diagrams we found that the sum of the two-loop leading and angulardependent next-to-leading logarithmic corrections can be written as the second-order term of a product of two exponential functions. The first exponential contains the part of the corrections corresponding to $\lambda=M$, i.e. the $\mathrm{SU}(2) \times \mathrm{U}(1)$ symmetric part. The second, outer exponential contains the contributions that originate from the mass gap $\lambda \ll M$ and corresponds to the QED corrections subtracted in such a way that they vanish at $\lambda=M$.

This result agrees with resummation prescriptions that have been proposed in the literature. These prescriptions are based on the assumption that, in the high-energy limit, the electroweak theory can be described by a symmetric $\mathrm{SU}(2) \times \mathrm{U}(1)$ theory matched with QED at the electroweak scale, and that no additional effects from spontaneous symmetry breaking appear. Our result, which has been derived within the spontaneously broken phase of the electroweak theory and in the physical basis, demonstrates that this assumption is correct at the next-to-leading angular-dependent logarithmic level.

Our derivations depend only on a few general features of the Electroweak Standard Model, i.e. on the underlying gauge symmetry and the fact that all particle masses are of the order of the electroweak scale or lighter. Therefore our result is also valid for those extensions of the Electroweak Standard Model that contain only novel particles with masses of the order of the weak scale and no additional gauge bosons. Such models include, for instance, the Electroweak Standard Model with two Higgs doublets or softly broken supersymmetric extensions such as the Minimal Supersymmetric Standard Model in the case where the masses of the Higgs bosons and the superpartner particles are of the order of the electroweak scale. 


\section{Acknowledgements}

This work was supported in part by the Swiss Bundesamt für Bildung und Wissenschaft and by the European Union under contract HPRN-CT-2000-00149. We thank Stefan Dittmaier for carefully reading the manuscript.

\section{A Gauge-group generators}

All our derivations are performed in terms of the physical (mass-eigenstate) gauge bosons $\gamma, \mathrm{Z}, \mathrm{W}^{ \pm}$. The corresponding gauge couplings result from combinations of the generators $T^{a}$ and $Y$ of the electroweak $\mathrm{SU}(2) \times \mathrm{U}(1)$ gauge group, and read

$$
I^{\gamma}=-Q, \quad I^{Z}=\frac{T^{3}-s_{\mathrm{W}}^{2} Q}{s_{\mathrm{W}} c_{\mathrm{W}}}, \quad I^{W^{ \pm}}=\frac{1}{s_{\mathrm{W}}} T^{ \pm}=\frac{1}{s_{\mathrm{W}}} \frac{T^{1} \pm \mathrm{i} T^{2}}{\sqrt{2}},
$$

where $Q=T^{3}+Y / 2$ represents the electric charge, and we use the shorthands $c_{\mathrm{w}}=\cos \theta_{\mathrm{w}}$ and $s_{\mathrm{W}}=\sin \theta_{\mathrm{w}}$ for the weak mixing angle, which is fixed by $c_{\mathrm{w}}^{2}=1-s_{\mathrm{w}}^{2}=M_{\mathrm{W}}^{2} / M_{\mathrm{Z}}^{2}$ in the on-shell renormalization scheme.

For the matrix components of the generators (A.1) we use the notation $I_{i^{\prime} i}^{a}$, where $a=\gamma, Z, W^{ \pm}$denotes the gauge fields, whereas the indices $i^{\prime}$ and $i$ correspond to two physical (mass-eigenstate) components $\varphi_{i^{\prime}}$ and $\varphi_{i}$ of a multiplet. The explicit matrix representations corresponding to the scalar doublet, right- or left-handed fermions and gauge bosons, as well as more details concerning our conventions, can be found in App. B of Ref. [17].

The matrix component $I_{i^{\prime} i}^{a}$ determines the gauge coupling for the vertex with the particles $V^{a}$ and $\varphi_{i}$ incoming and the particle $\varphi_{i^{\prime}}$ outgoing. The fields and the matrix components are in general complex and satisfy the relations [17]

$$
\left(I_{j i}^{a}\right)^{*}=-I_{\bar{j} \bar{i}}^{\bar{a}}, \quad I_{i j}^{a}=-I_{\bar{j} i}^{a},
$$

where the particles $\bar{a}, \bar{i}, \ldots$ correspond to the charge conjugated of $a, i, \ldots$.

In our derivations we make extensive use of the commutation relations

$$
\left[I^{a}, I^{b}\right]=\sum_{c=\gamma, Z, W^{ \pm}} I^{c} I_{c b}^{a}
$$

and of the well-known commutation relations

$$
\sum_{a=\gamma, Z, W^{ \pm}}\left[I^{a} I^{\bar{a}}, I^{b}\right]=0 \quad \text { with } \quad b=\gamma, Z, W^{ \pm},
$$

for the electroweak Casimir operator

$$
C^{\mathrm{ew}}:=\sum_{a=\gamma, Z, W^{ \pm}} I^{a} I^{\bar{a}}=\frac{1}{c_{\mathrm{W}}^{2}}\left(\frac{Y}{2}\right)^{2}+\frac{1}{s_{\mathrm{W}}^{2}} T(T+1),
$$

where $T$ is the total isospin, and $T(T+1)$ is the Casimir operator of the $\mathrm{SU}(2)$ group. The electroweak Casimir operator is a diagonal matrix apart from the neutral gauge-boson sector, where mixing gives rise to the non-diagonal components $C_{\gamma Z}^{\mathrm{ew}}=C_{Z \gamma}^{\mathrm{ew}}=-2 c_{\mathrm{W}} / s_{\mathrm{W}}$ (see App. B of Ref. [17]). 


\section{B 1- and 2-loop integrals in logarithmic approximation}

In this section we present detailed results for the one- and two-loop integrals involving soft-collinear gauge bosons. For each diagram we first specify the corresponding Feynman integral in eikonal approximation (eik.). Then we also give the corresponding integral in the Sudakov approximation (Sud.). Finally, we present explicit results in next-to-leading logarithmic angular-dependent $\left(\mathrm{NLL}_{\mathrm{a}}\right)$ approximation (2.4), which have been obtained in the high-energy limit (2.3) and for all cases specified in (2.11) and (2.12). These results were derived using the Sudakov approximation and the sector-decomposition method described in Sect. 3.1 and Sect. 3.2, respectively.

The external momenta are assumed to be on-shell, $p_{k}^{2}=m_{k}^{2}$, with masses at or below the electroweak scale, i.e. $M \gtrsim m_{k} \gg \lambda$. Masses that do not regularize mass singularities are neglected. Consequently, the masses of the external particles and of the internal particles that are not soft-collinear gauge bosons are only relevant for photon exchange diagrams, where the masses before and after photon emission are equal. Therefore, we can set the internal and external masses of the particle lines equal in the following.

\section{B.1 One-loop integrals}

For the one-loop diagram

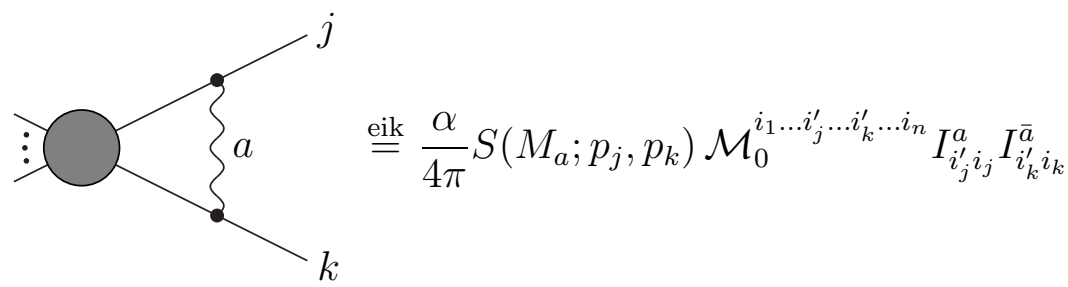

we have the integral

$$
S\left(M_{a} ; p_{j}, p_{k}\right):=-\mathrm{i}(4 \pi)^{2} \int \frac{\mathrm{d}^{4} l_{1}}{(2 \pi)^{4}} \frac{4 p_{j} p_{k}}{\left[l_{1}^{2}-M_{a}^{2}\right]\left[\left(p_{j}-l_{1}\right)^{2}-m_{j}^{2}\right]\left[\left(p_{k}+l_{1}\right)-m_{k}^{2}\right]} .
$$

Here and in the following we suppress the infinitesimal imaginary parts i $\varepsilon$ of the causal propagators for brevity. In the Sudakov approximation

$$
\begin{aligned}
& S\left(M_{a} ; p_{j}, p_{k}\right) \stackrel{\text { Sud }}{=} \\
& \quad=2 \int_{0}^{1} \frac{\mathrm{d} x_{1}}{x_{1}} \int_{0}^{1} \frac{\mathrm{d} y_{1}}{y_{1}} \theta\left(x_{1} y_{1}-\frac{M_{a}^{2}}{\left|2 p_{j} p_{k}\right|}\right) \theta\left(y_{1}-\frac{m_{j}^{2}}{\left|2 p_{j} p_{k}\right|} x_{1}\right) \theta\left(x_{1}-\frac{m_{k}^{2}}{\left|2 p_{j} p_{k}\right|} y_{1}\right) .
\end{aligned}
$$

The results in $\mathrm{NLL}_{\mathrm{a}}$ approximation corresponding to the cases $M_{a}=M, \lambda$, are given in (4.3) and (4.4). 


\section{B.2 Two-loop integrals}

2-leg ladder diagram $S_{2 \mathrm{~L}}$

We begin with the planar ladder diagram

$$
D_{2 \mathrm{~L}, j k}^{a b}:=2_{k}\left\{\begin{array}{l}
j \stackrel{\text { eik }}{=}\left(\frac{\alpha}{4 \pi}\right)^{2} S_{2 \mathrm{~L}}\left(M_{a}, M_{b} ; p_{j}, p_{k}\right) \\
\times \mathcal{M}_{0}^{i_{1} \ldots i_{j}^{\prime} \ldots i_{k}^{\prime} \ldots i_{n}}\left(I^{b} I^{a}\right)_{i_{j}^{\prime} i_{j}}\left(I^{\bar{b}} I^{\bar{a}}\right)_{i_{k}^{\prime} i_{k}}
\end{array}\right.
$$

with

$$
\begin{aligned}
& S_{2 \mathrm{~L}}(\left.M_{a}, M_{b} ; p_{j}, p_{k}\right):=-(4 \pi)^{4} \int \frac{\mathrm{d}^{4} l_{1}}{(2 \pi)^{4}} \int \frac{\mathrm{d}^{4} l_{2}}{(2 \pi)^{4}} \frac{1}{\left[l_{1}^{2}-M_{a}^{2}\right]\left[\left(p_{j}-l_{1}\right)^{2}-m_{j}^{2}\right]} \\
& \times \frac{16\left(p_{j} p_{k}\right)^{2}}{\left[\left(p_{k}+l_{1}\right)^{2}-m_{k}^{2}\right]\left[l_{2}^{2}-M_{b}^{2}\right]\left[\left(p_{j}-l_{1}-l_{2}\right)^{2}-m_{j}^{2}\right]\left[\left(p_{k}+l_{1}+l_{2}\right)^{2}-m_{k}^{2}\right]} .
\end{aligned}
$$

In the Sudakov approximation

$$
\begin{aligned}
S_{2 \mathrm{~L}}( & \left.M_{a}, M_{b} ; p_{j}, p_{k}\right) \stackrel{\mathrm{Sud}}{=} \\
= & 4 \int_{0}^{1} \frac{\mathrm{d} x_{1}}{x_{1}} \int_{0}^{1} \frac{\mathrm{d} y_{1}}{y_{1}} \int_{0}^{1} \frac{\mathrm{d} x_{2}}{x_{2}} \int_{0}^{1} \frac{\mathrm{d} y_{2}}{y_{2}} \theta\left(x_{1} y_{1}-\frac{M_{a}^{2}}{\left|2 p_{j} p_{k}\right|}\right) \theta\left(x_{2} y_{2}-\frac{M_{b}^{2}}{\left|2 p_{j} p_{k}\right|}\right) \\
& \times \theta\left(x_{2}-x_{1}\right) \theta\left(y_{2}-y_{1}\right) \theta\left(x_{1}-\frac{m_{j}^{2}}{\left|2 p_{j} p_{k}\right|} y_{1}\right) \theta\left(y_{1}-\frac{m_{k}^{2}}{\left|2 p_{j} p_{k}\right|} x_{1}\right) \\
& \times \theta\left(x_{2}-\frac{m_{j}^{2}}{\left|2 p_{j} p_{k}\right|} y_{2}\right) \theta\left(y_{2}-\frac{m_{k}^{2}}{\left|2 p_{j} p_{k}\right|} x_{2}\right) .
\end{aligned}
$$

In $\mathrm{NLL}_{\mathrm{a}}$ approximation, we find the following expressions for the cases (2.11):

$$
\begin{aligned}
& S_{2 \mathrm{~L}}(\left.\lambda, \lambda ; p_{j}, p_{k}\right) \stackrel{\mathrm{NLL}}{=} \frac{1}{2} \log ^{2} \frac{\lambda^{2}}{\left|2 p_{j} p_{k}\right|} \log ^{2} \frac{m_{j}^{2} m_{k}^{2}}{\left(2 p_{j} p_{k}\right)^{2}}-\frac{1}{6} \log \frac{\lambda^{2}}{\left|2 p_{j} p_{k}\right|} \\
& \times {\left[2 \log ^{3} \frac{m_{j}^{2} m_{k}^{2}}{\left(2 p_{j} p_{k}\right)^{2}}+3 \log \frac{m_{j}^{2} m_{k}^{2}}{\left(2 p_{j} p_{k}\right)^{2}}\left(\log ^{2} \frac{m_{j}^{2}}{\left|2 p_{j} p_{k}\right|}+\log ^{2} \frac{m_{k}^{2}}{\left|2 p_{j} p_{k}\right|}\right)\right] } \\
&+ \frac{5}{12}\left[\log ^{4} \frac{m_{j}^{2}}{\left|2 p_{j} p_{k}\right|}+\log ^{4} \frac{m_{k}^{2}}{\left|2 p_{j} p_{k}\right|}\right]+\log ^{2} \frac{m_{j}^{2}}{\left|2 p_{j} p_{k}\right|} \log ^{2} \frac{m_{k}^{2}}{\left|2 p_{j} p_{k}\right|} \\
&+ \frac{5}{6} \log \frac{m_{j}^{2}}{\left|2 p_{j} p_{k}\right|} \log \frac{m_{k}^{2}}{\left|2 p_{j} p_{k}\right|}\left(\log ^{2} \frac{m_{j}^{2}}{\left|2 p_{j} p_{k}\right|}+\log ^{2} \frac{m_{k}^{2}}{\left|2 p_{j} p_{k}\right|}\right), \\
& S_{2 \mathrm{~L}}\left(\lambda, M ; p_{j}, p_{k}\right) \stackrel{\mathrm{NLLa}}{=}-\frac{1}{6} \log ^{4} \frac{M^{2}}{\left|2 p_{j} p_{k}\right|}-\frac{2}{3} \log ^{3} \frac{M^{2}}{\left|2 p_{j} p_{k}\right|} \log \frac{m_{j}^{2} m_{k}^{2}}{\left(2 p_{j} p_{k}\right)^{2}} \\
&-\frac{1}{2} \log ^{2} \frac{M^{2}}{\left|2 p_{j} p_{k}\right|}\left[\log ^{2} \frac{m_{j}^{2}}{\left|2 p_{j} p_{k}\right|}+\log ^{2} \frac{m_{k}^{2}}{\left|2 p_{j} p_{k}\right|}-2 \log \frac{\lambda^{2}}{\left|2 p_{j} p_{k}\right|} \log \frac{m_{j}^{2} m_{k}^{2}}{\left(2 p_{j} p_{k}\right)^{2}}\right], \\
& S_{2 \mathrm{~L}}\left(M, \lambda ; p_{j}, p_{k}\right) \stackrel{\mathrm{NLL}_{\mathrm{a}}}{=} S_{2 \mathrm{~L}}\left(M, M ; p_{j}, p_{k}\right) \stackrel{\mathrm{NLL}_{\mathrm{a}}}{=} \frac{1}{6} \log ^{4} \frac{M^{2}}{\left|2 p_{j} p_{k}\right|} .
\end{aligned}
$$


2-leg crossed ladder diagram $S_{2 \mathrm{C}}$

For the 2-leg crossed (non-planar) ladder diagram

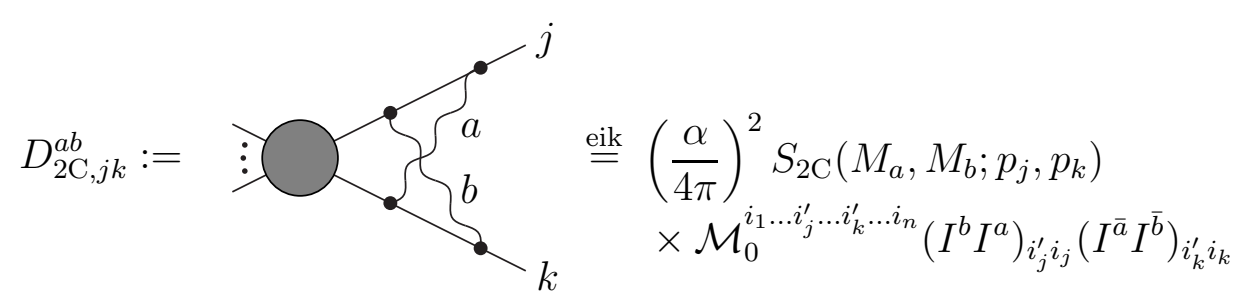

we have

$$
\begin{aligned}
& S_{2 \mathrm{C}}(\left.M_{a}, M_{b} ; p_{j}, p_{k}\right):=-(4 \pi)^{4} \int \frac{\mathrm{d}^{4} l_{1}}{(2 \pi)^{4}} \int \frac{\mathrm{d}^{4} l_{2}}{(2 \pi)^{4}} \frac{1}{\left[l_{1}^{2}-M_{a}^{2}\right]\left[\left(p_{j}-l_{1}\right)^{2}-m_{j}^{2}\right]} \\
& \times \frac{16\left[p_{j}\left(p_{k}-l_{2}\right)\right]\left[p_{k}\left(p_{j}-l_{1}\right)\right]}{\left[l_{2}^{2}-M_{b}^{2}\right]\left[\left(p_{k}-l_{2}\right)^{2}-m_{k}^{2}\right]\left[\left(p_{j}-l_{1}+l_{2}\right)^{2}-m_{j}^{2}\right]\left[\left(p_{k}+l_{1}-l_{2}\right)^{2}-m_{k}^{2}\right]} .
\end{aligned}
$$

As discussed in Sect. 2.2, in order to avoid spurious leading logarithms originating from the region $l_{1} \approx p_{j}$ and $l_{2} \approx p_{k}$ when the integral is evaluated in the Feynman-parameter representation, loop-momentum-dependent eikonal factors (2.10) have to be used for the inner vertices of this topology.

In the Sudakov approximation, where the loop-momentum dependence of the eikonal factors can be neglected, we have

$$
\begin{aligned}
S_{2 \mathrm{C}}( & \left.M_{a}, M_{b} ; p_{j}, p_{k}\right) \stackrel{\text { Sud }}{=} \\
= & 4 \int_{0}^{1} \frac{\mathrm{d} x_{1}}{x_{1}} \int_{0}^{1} \frac{\mathrm{d} y_{1}}{y_{1}} \int_{0}^{1} \frac{\mathrm{d} x_{2}}{x_{2}} \int_{0}^{1} \frac{\mathrm{d} y_{2}}{y_{2}} \theta\left(x_{1} y_{1}-\frac{M_{a}^{2}}{\left|2 p_{j} p_{k}\right|}\right) \theta\left(x_{2} y_{2}-\frac{M_{b}^{2}}{\left|2 p_{j} p_{k}\right|}\right) \\
& \times \theta\left(y_{1}-\frac{m_{j}^{2}}{\left|2 p_{j} p_{k}\right|} x_{1}\right) \theta\left(x_{2}-\frac{m_{k}^{2}}{\left|2 p_{j} p_{k}\right|} y_{2}\right) \theta\left(y_{2}-y_{1}\right) \theta\left(x_{1}-x_{2}\right) .
\end{aligned}
$$

In $\mathrm{NLL}_{\mathrm{a}}$ approximation, we derive the following expressions for the cases (2.11):

$$
\begin{aligned}
& S_{2 \mathrm{C}}\left(\lambda, \lambda ; p_{j}, p_{k}\right) \stackrel{\mathrm{NLL}}{=} \frac{1}{3} \log \frac{\lambda^{2}}{\left|2 p_{j} p_{k}\right|} \log ^{3} \frac{m_{j}^{2} m_{k}^{2}}{\left(2 p_{j} p_{k}\right)^{2}} \\
& \quad-\frac{7}{24}\left(\log ^{4} \frac{m_{j}^{2}}{\left|2 p_{j} p_{k}\right|}+\log ^{4} \frac{m_{j}^{2}}{\left|2 p_{j} p_{k}\right|}\right)-\frac{3}{4} \log ^{2} \frac{m_{j}^{2}}{\left|2 p_{j} p_{k}\right|} \log ^{2} \frac{m_{k}^{2}}{\left|2 p_{j} p_{k}\right|} \\
& \quad-\frac{5}{6} \log \frac{m_{j}^{2}}{\left|2 p_{j} p_{k}\right|} \log \frac{m_{k}^{2}}{\left|2 p_{j} p_{k}\right|}\left[\log ^{2} \frac{m_{j}^{2}}{\left|2 p_{j} p_{k}\right|}+\log ^{2} \frac{m_{k}^{2}}{\left|2 p_{j} p_{k}\right|}\right] \\
& S_{2 \mathrm{C}}\left(\lambda, M ; p_{j}, p_{k}\right)=S_{2 \mathrm{C}}\left(M, \lambda ; p_{k}, p_{j}\right) \stackrel{\mathrm{NLL}_{\mathrm{a}}}{\frac{2}{3}} \log ^{3} \frac{M^{2}}{\left|2 p_{j} p_{k}\right|} \log \frac{m_{j}^{2}}{\left|2 p_{j} p_{k}\right|} \\
& S_{2 \mathrm{C}}\left(M, M ; p_{j}, p_{k}\right) \stackrel{\mathrm{NLLa}}{=} \frac{1}{3} \log ^{4} \frac{M^{2}}{\left|2 p_{j} p_{k}\right|} .
\end{aligned}
$$


2-leg Yang-Mills diagram $S_{2 \mathrm{Y}}$

For the 2-leg Yang-Mills diagram

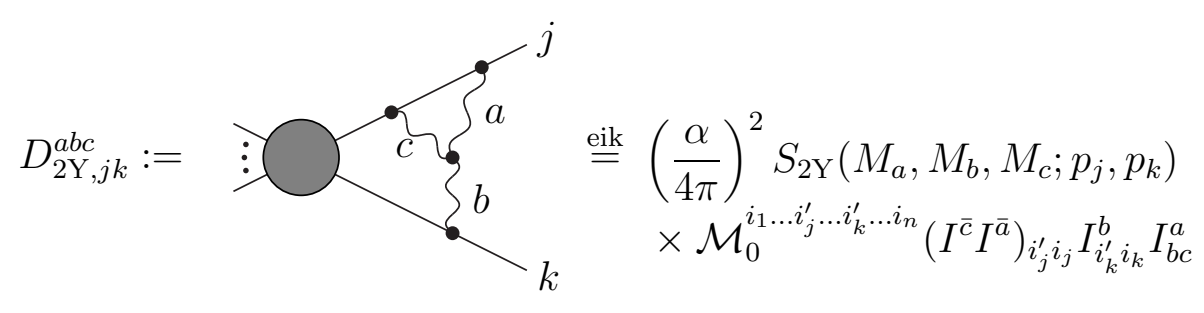

we have

$$
\begin{gathered}
S_{2 \mathrm{Y}}\left(M_{a}, M_{b}, M_{c} ; p_{j}, p_{k}\right):=(4 \pi)^{4} \int \frac{\mathrm{d}^{4} l_{1}}{(2 \pi)^{4}} \int \frac{\mathrm{d}^{4} l_{2}}{(2 \pi)^{4}} \frac{1}{\left[l_{1}^{2}-M_{a}^{2}\right]\left[\left(p_{j}-l_{1}\right)^{2}-m_{j}^{2}\right]} \\
\times \frac{8 p_{j} p_{k}\left[\left(l_{2}+2 l_{1}\right) p_{j}\right]}{\left[l_{2}^{2}-M_{b}^{2}\right]\left[\left(l_{1}+l_{2}\right)^{2}-M_{c}^{2}\right]\left[\left(p_{j}+l_{2}\right)^{2}-m_{j}^{2}\right]\left[\left(p_{k}-l_{2}\right)^{2}-m_{k}^{2}\right]} .
\end{gathered}
$$

For the cases (2.12) we find the Sudakov approximation

$$
\begin{aligned}
S_{2 \mathrm{Y}}( & \left.M_{a}, M_{b}, M_{c} ; p_{j}, p_{k}\right) \stackrel{\text { Sud }}{=} \\
= & 2 \int_{0}^{1} \frac{\mathrm{d} x_{1}}{x_{1}} \int_{0}^{1} \frac{\mathrm{d} y_{1}}{y_{1}} \int_{0}^{1} \frac{\mathrm{d} x_{2}}{x_{2}} \int_{0}^{1} \frac{\mathrm{d} y_{2}}{y_{2}} \theta\left(x_{1} y_{2}-x_{2} y_{1}\right) \theta\left(y_{1}-\frac{m_{j}^{2}}{\left|2 p_{j} p_{k}\right|} x_{1}\right) \\
& \times \theta\left(y_{2}-\frac{m_{j}^{2}}{\left|2 p_{j} p_{k}\right|} x_{2}\right) \theta\left(x_{2}-\frac{m_{k}^{2}}{\left|2 p_{j} p_{k}\right|} y_{2}\right) \\
& \times\left\{\theta\left(x_{1} y_{1}-\frac{M_{c}^{2}}{\left|2 p_{j} p_{k}\right|}\right) \theta\left(x_{2} y_{2}-\frac{M_{b}^{2}}{\left|2 p_{j} p_{k}\right|}\right) \theta\left(y_{1}-y_{2}\right)+\theta\left(x_{1} y_{1}-\frac{M_{a}^{2}}{\left|2 p_{j} p_{k}\right|}\right)\right. \\
& \times \theta\left(x_{2} y_{2}-\frac{M_{c}^{2}}{\left|2 p_{j} p_{k}\right|}\right) \theta\left(x_{2}-x_{1}\right) \theta\left(y_{2}-y_{1}\right)-\theta\left(x_{1} y_{1}-\frac{M_{a}^{2}}{\left|2 p_{j} p_{k}\right|}\right) \\
& \left.\times \theta\left(x_{2} y_{2}-\frac{M_{b}^{2}}{\left|2 p_{j} p_{k}\right|}\right) \theta\left(x_{1} y_{2}-\left|\frac{M_{c}^{2}}{\left|2 p_{j} p_{k}\right|}-\frac{M_{a}^{2}}{\left|2 p_{j} p_{k}\right|}-\frac{M_{b}^{2}}{\left|2 p_{j} p_{k}\right|}\right|\right)\right\},
\end{aligned}
$$

and in $\mathrm{NLL}_{\mathrm{a}}$ approximation we obtain

$$
\begin{aligned}
& S_{2 \mathrm{Y}}\left(\lambda, M, M ; p_{j}, p_{k}\right) \stackrel{\mathrm{NLL}_{\mathrm{a}}}{=} S_{2 \mathrm{Y}}\left(M, \lambda, M ; p_{k}, p_{j}\right) \stackrel{\mathrm{NLL}_{\mathrm{a}}}{=} \\
& \stackrel{\mathrm{NLL}_{a}}{=}-\frac{1}{3} \log ^{3} \frac{M^{2}}{\left|2 p_{j} p_{k}\right|} \log \frac{m_{j}^{2}}{\left|2 p_{j} p_{k}\right|}, \\
& S_{2 \mathrm{Y}}\left(M, M, \lambda ; p_{j}, p_{k}\right) \stackrel{\mathrm{NLLa}_{\mathrm{a}}}{=} S_{2 \mathrm{Y}}\left(M, M, M ; p_{j}, p_{k}\right) \stackrel{\mathrm{NLL}^{2}}{=}-\frac{1}{6} \log ^{4} \frac{M^{2}}{\left|2 p_{j} p_{k}\right|} .
\end{aligned}
$$


3-leg ladder diagram $S_{3 \mathrm{~L}}$

For the 3-leg ladder diagram

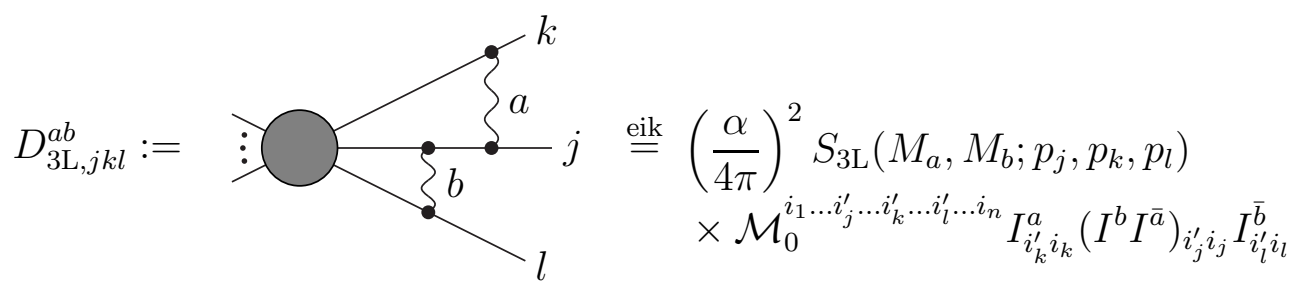

we have

$$
\begin{aligned}
S_{3 \mathrm{~L}} & \left(M_{a}, M_{b} ; p_{j}, p_{k}, p_{l}\right):=-(4 \pi)^{4} \int \frac{\mathrm{d}^{4} l_{1}}{(2 \pi)^{4}} \int \frac{\mathrm{d}^{4} l_{2}}{(2 \pi)^{4}} \frac{1}{\left[l_{1}^{2}-M_{a}^{2}\right]\left[\left(p_{j}+l_{1}\right)^{2}-m_{j}^{2}\right]} \\
& \times \frac{16\left(p_{j} p_{k}\right)\left[\left(p_{j}+l_{1}\right) p_{l}\right]}{\left[\left(p_{k}-l_{1}\right)^{2}-m_{k}^{2}\right]\left[l_{2}^{2}-M_{b}^{2}\right]\left[\left(p_{j}+l_{1}+l_{2}\right)^{2}-m_{j}^{2}\right]\left[\left(p_{l}-l_{2}\right)^{2}-m_{l}^{2}\right]} .
\end{aligned}
$$

As discussed in Sect. 2.2, in order to avoid spurious leading logarithms originating from the region $l_{1} \approx-p_{j}$ and $l_{2} \approx 0$ when the integral is evaluated in the Feynman-parameter representation, a loop-momentum-dependent eikonal factor (2.10) has to be used for the emission of the gauge boson $V^{b}$ along the line $j$ in this topology.

In the Sudakov approximation, where the loop-momentum dependence of the eikonal factor can be neglected, we have

$$
\begin{aligned}
S_{3 \mathrm{~L}}( & \left.M_{a}, M_{b} ; p_{j}, p_{k}, p_{l}\right) \stackrel{\text { Sud }}{=} \\
= & 4 \int_{0}^{1} \frac{\mathrm{d} x_{1}}{x_{1}} \int_{0}^{1} \frac{\mathrm{d} y_{1}}{y_{1}} \int_{0}^{1} \frac{\mathrm{d} x_{2}}{x_{2}} \int_{0}^{1} \frac{\mathrm{d} y_{2}}{y_{2}} \theta\left(x_{1} y_{1}-\frac{M_{a}^{2}}{\left|2 p_{j} p_{k}\right|}\right) \theta\left(x_{2} y_{2}-\frac{M_{b}^{2}}{\left|2 p_{j} p_{l}\right|}\right) \\
& \times \theta\left(x_{2}-\frac{\left|p_{j} p_{k}\right|}{\left|p_{j} p_{l}\right|} x_{1}\right) \theta\left(x_{1}-\frac{m_{j}^{2}}{\left|2 p_{j} p_{k}\right|} y_{1}\right) \theta\left(x_{2}-\frac{m_{j}^{2}}{\left|2 p_{j} p_{l}\right|} y_{2}\right) \\
& \times \theta\left(y_{1}-\frac{m_{k}^{2}}{\left|2 p_{j} p_{k}\right|} x_{1}\right) \theta\left(y_{2}-\frac{m_{l}^{2}}{\left|2 p_{j} p_{l}\right|} x_{2}\right) .
\end{aligned}
$$

Neglecting angular-dependent NNLL of order $\log ^{2}\left(s / M^{2}\right) \log ^{2}\left(2 p_{m} p_{n} / s\right)$, we obtain the following results for the cases (2.11) in $\mathrm{NLL}_{\mathrm{a}}$ approximation:

$$
\begin{aligned}
S_{3 \mathrm{~L}}( & \left.\lambda, \lambda ; p_{j}, p_{k}, p_{l}\right) \stackrel{\text { NLLa }}{=} \frac{1}{2} \log \frac{m_{j}^{2} m_{k}^{2}}{\left(2 p_{j} p_{k}\right)^{2}} \log \frac{m_{j}^{2} m_{l}^{2}}{\left(2 p_{j} p_{l}\right)^{2}} \log ^{2} \frac{\lambda^{2}}{\left|2 p_{j} p_{k}\right|} \\
- & \frac{1}{2} \log \frac{m_{j}^{2} m_{k}^{2}}{\left(2 p_{j} p_{k}\right)^{2}} \log \frac{\lambda^{2}}{\left|2 p_{j} p_{k}\right|}\left[\log ^{2} \frac{m_{j}^{2}}{\left|2 p_{j} p_{l}\right|}+\log ^{2} \frac{m_{l}^{2}}{\left|2 p_{j} p_{l}\right|}\right. \\
& \left.+\log \frac{m_{j}^{2} m_{l}^{2}}{\left(2 p_{j} p_{l}\right)^{2}}\left(\log \frac{m_{k}^{2}}{m_{l}^{2}}-4 \log \frac{\left|p_{j} p_{k}\right|}{\left|p_{j} p_{l}\right|}\right)\right] \\
+ & \frac{1}{8}\left[\log ^{2} \frac{m_{j}^{2}}{\left|2 p_{j} p_{l}\right|}+\log ^{2} \frac{m_{l}^{2}}{\left|2 p_{j} p_{l}\right|}\right]\left[\log ^{2} \frac{m_{j}^{2}}{\left|2 p_{j} p_{k}\right|}+\log ^{2} \frac{m_{k}^{2}}{\left|2 p_{j} p_{k}\right|}\right] \\
+ & \log ^{3} \frac{m_{j}^{2}}{\left|2 p_{j} p_{k}\right|}\left[\frac{2}{3} \log \frac{m_{k}^{2}}{m_{l}^{2}}-\frac{19}{12} \log \frac{\left|p_{j} p_{k}\right|}{\left|p_{j} p_{l}\right|}\right]
\end{aligned}
$$




$$
\begin{aligned}
& +\log ^{2} \frac{m_{j}^{2}}{\left|2 p_{j} p_{k}\right|}\left[\frac{3}{8} \log ^{2} \frac{m_{k}^{2}}{\left|2 p_{j} p_{k}\right|}-\frac{3}{8} \log ^{2} \frac{m_{l}^{2}}{\left|2 p_{j} p_{k}\right|}-\frac{9}{4} \log \frac{\left|p_{j} p_{k}\right|}{\left|p_{j} p_{l}\right|} \log \frac{m_{l}^{2}}{\left|2 p_{j} p_{k}\right|}\right] \\
& +\log \frac{m_{j}^{2}}{\left|2 p_{j} p_{k}\right|}\left[\frac{1}{6} \log ^{3} \frac{m_{k}^{2}}{\left|2 p_{j} p_{k}\right|}-\frac{1}{6} \log ^{3} \frac{m_{l}^{2}}{\left|2 p_{j} p_{k}\right|}\right. \\
& \left.-\log \frac{\left|p_{j} p_{k}\right|}{\left|p_{j} p_{l}\right|}\left(\frac{1}{4} \log ^{2} \frac{m_{k}^{2}}{\left|2 p_{j} p_{k}\right|}+\log ^{2} \frac{m_{l}^{2}}{\left|2 p_{j} p_{k}\right|}\right)\right] \\
& -\frac{1}{24} \log ^{4} \frac{m_{l}^{2}}{\left|2 p_{j} p_{k}\right|}+\frac{1}{6} \log ^{3} \frac{m_{k}^{2}}{\left|2 p_{j} p_{k}\right|} \log \frac{m_{l}^{2}}{\left|2 p_{j} p_{k}\right|}-\frac{1}{8} \log ^{2} \frac{m_{k}^{2}}{\left|2 p_{j} p_{k}\right|} \log ^{2} \frac{m_{l}^{2}}{\left|2 p_{j} p_{k}\right|} \\
& +\log \frac{\left|p_{j} p_{k}\right|}{\left|p_{j} p_{l}\right|}\left(\frac{1}{3} \log ^{3} \frac{m_{k}^{2}}{\left|2 p_{j} p_{k}\right|}-\frac{5}{4} \log ^{2} \frac{m_{k}^{2}}{\left|2 p_{j} p_{k}\right|} \log \frac{m_{l}^{2}}{\left|2 p_{j} p_{k}\right|}-\frac{1}{3} \log ^{3} \frac{m_{l}^{2}}{\left|2 p_{j} p_{k}\right|}\right) \\
& +\theta\left(m_{k}\left(p_{j} p_{l}\right)^{2}-m_{l}\left(p_{j} p_{k}\right)^{2}\right)\left(\frac{1}{24} \log ^{4} \frac{m_{l}^{2}}{m_{k}^{2}}+\frac{1}{3} \log \frac{\left|p_{j} p_{k}\right|}{\left|p_{j} p_{l}\right|} \log ^{3} \frac{m_{l}^{2}}{m_{k}^{2}}\right), \\
& S_{3 \mathrm{~L}}\left(M, \lambda ; p_{j}, p_{k}, p_{l}\right) \stackrel{\mathrm{NLL}_{\mathrm{a}}}{=}\left[\frac{2}{3} \log \frac{m_{l}^{2}}{\left|2 p_{j} p_{l}\right|}+\frac{1}{6} \log \frac{M^{2}}{\left|2 p_{j} p_{l}\right|}\right] \log ^{3} \frac{M^{2}}{\left|2 p_{j} p_{l}\right|}, \\
& S_{3 \mathrm{~L}}\left(\lambda, M ; p_{j}, p_{k}, p_{l}\right) \stackrel{\mathrm{NLL}_{\mathrm{a}}}{=} \log \frac{\lambda^{2}}{\left|2 p_{j} p_{k}\right|} \log \frac{m_{j}^{2} m_{k}^{2}}{\left(2 p_{j} p_{k}\right)^{2}} \log ^{2} \frac{M^{2}}{\left|2 p_{j} p_{l}\right|}-\frac{1}{6} \log ^{4} \frac{M^{2}}{\left|2 p_{j} p_{k}\right|} \\
& -\frac{1}{2} \log ^{2} \frac{M^{2}}{\left|2 p_{j} p_{l}\right|}\left(\log ^{2} \frac{m_{j}^{2}}{\left|2 p_{j} p_{k}\right|}+\log ^{2} \frac{m_{k}^{2}}{\left|2 p_{j} p_{k}\right|}\right) \\
& -\frac{2}{3} \log ^{3} \frac{M^{2}}{\left|2 p_{j} p_{k}\right|} \log \frac{m_{k}^{2}}{\left|2 p_{j} p_{k}\right|} \\
& S_{3 \mathrm{~L}}\left(M, M ; p_{j}, p_{k}, p_{l}\right) \stackrel{\mathrm{NLL}_{\mathrm{a}}}{=} \frac{1}{2} \log ^{4} \frac{M^{2}}{\left|2 p_{j} p_{l}\right|} \text {. }
\end{aligned}
$$

\section{3-leg Yang-Mills diagram $S_{3 \mathrm{Y}}$}

For the 3-leg Yang-Mills diagram

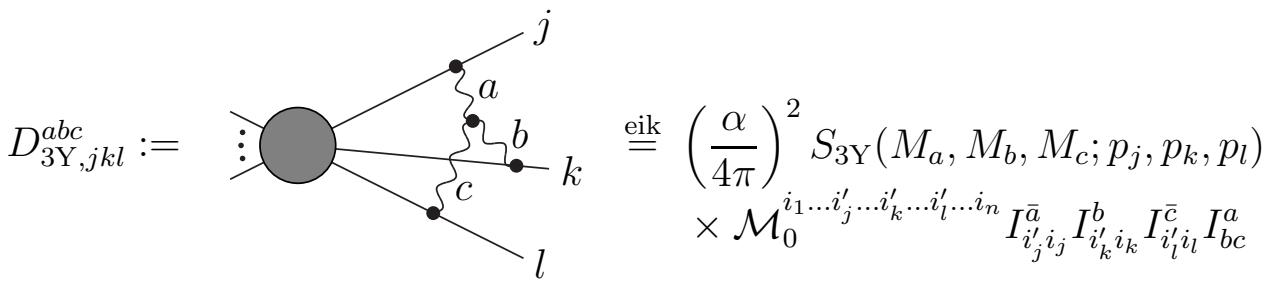

we have

$$
\begin{aligned}
S_{3 \mathrm{Y}}\left(M_{a}, M_{b}, M_{c} ; p_{j}, p_{k}, p_{l}\right):=(4 \pi)^{4} \int \frac{\mathrm{d}^{4} l_{1}}{(2 \pi)^{4}} \int \frac{\mathrm{d}^{4} l_{2}}{(2 \pi)^{4}} \frac{1}{\left[l_{1}^{2}-M_{a}^{2}\right]\left[\left(p_{j}-l_{1}\right)^{2}-m_{j}^{2}\right]} \\
\times \frac{8 p_{k} p_{l}\left[\left(l_{1}+2 l_{2}\right) p_{j}\right]-8 p_{j} p_{k}\left[\left(l_{2}-l_{1}\right) p_{l}\right]-8 p_{j} p_{l}\left[\left(2 l_{1}+l_{2}\right) p_{k}\right]}{\left[l_{2}^{2}-M_{b}^{2}\right]\left[\left(l_{1}+l_{2}\right)^{2}-M_{c}^{2}\right]\left[\left(p_{k}-l_{2}\right)^{2}-m_{k}^{2}\right]\left[\left(p_{l}+l_{1}+l_{2}\right)^{2}-m_{l}^{2}\right]} .
\end{aligned}
$$

Neglecting angular-dependent NNLL of order $\log ^{2}\left(s / M^{2}\right) \log ^{2}\left(2 p_{m} p_{n} / s\right)$, we obtain the following results for the cases (2.12) in $\mathrm{NLL}_{\mathrm{a}}$ approximation:

$$
S_{3 \mathrm{Y}}\left(\lambda, M, M ; p_{j}, p_{k}, p_{l}\right)=S_{3 \mathrm{Y}}\left(M, \lambda, M ; p_{l}, p_{j}, p_{k}\right)=S_{3 \mathrm{Y}}\left(M, M, \lambda ; p_{k}, p_{l}, p_{j}\right)
$$




$$
\begin{aligned}
& \stackrel{\mathrm{NLL}_{\mathrm{a}}}{=}-\frac{1}{3} \log \frac{\left|p_{j} p_{k}\right|}{\left|p_{j} p_{l}\right|} \log ^{2} \frac{M^{2}}{\left|2 p_{k} p_{l}\right|}\left[\log \frac{M^{2}}{\left|2 p_{k} p_{l}\right|}-3 \log \frac{m_{j}^{2}}{\left|2 p_{k} p_{l}\right|}\right], \\
& S_{3 \mathrm{Y}}\left(M, M, M ; p_{j}, p_{k}, p_{l}\right) \stackrel{\mathrm{NLL}_{\mathrm{a}}}{=} 0 .
\end{aligned}
$$

4-leg ladder diagram $S_{4 \mathrm{~L}}$

Finally, for the 4-leg ladder diagram

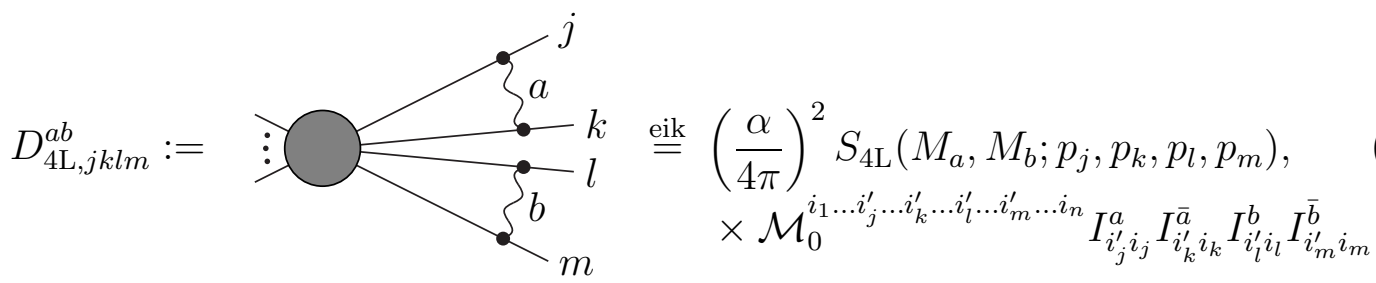

we have

$$
S_{4 \mathrm{~L}}\left(M_{a}, M_{b} ; p_{j}, p_{k}, p_{l}, p_{m}\right)=S\left(M_{a} ; p_{j}, p_{k}\right) S\left(M_{b} ; p_{l}, p_{m}\right)
$$

\section{Relations between loop integrals in logarithmic approximation}

In the following we list the relations between the one- and two-loop integrals that have been used in Sect. 4.2 in order to simplify the sum over all eikonal contributions. These relations have been obtained from the results of App. B.1 and App. B.2, and are valid in $\mathrm{NLL}_{\mathrm{a}}$ approximation${ }^{7}$ (2.4).

Combinations of 2-leg ladder integrals can be expressed as products of one-loop integrals using

$$
\left[S_{2 \mathrm{~L}}\left(M_{a}, M_{b} ; p_{j}, p_{k}\right)+S_{2 \mathrm{C}}\left(M_{a}, M_{b} ; p_{j}, p_{k}\right)\right]+(a \leftrightarrow b) \stackrel{\mathrm{NLL}_{\mathrm{a}}}{=} S\left(M_{a} ; p_{j}, p_{k}\right) S\left(M_{b} ; p_{j}, p_{k}\right),
$$

which is valid for all cases (2.11). The 2-leg Yang-Mills diagram can be related to the 2-leg crossed ladder diagram using

$$
S_{2 \mathrm{Y}}\left(M_{a}, M_{b}, M_{c} ; p_{j}, p_{k}\right) \stackrel{\mathrm{NLL}_{a}}{=}-\frac{1}{2} S_{2 \mathrm{C}}\left(M_{a}, M_{b} ; p_{j}, p_{k}\right)
$$

which is valid in all cases (2.12). Furthermore, we have

$$
\begin{aligned}
& S_{2 \mathrm{~L}}\left(M, \lambda ; p_{j}, p_{k}\right) \stackrel{\mathrm{NLL}_{\mathrm{a}}}{=} S_{2 \mathrm{~L}}\left(M, M ; p_{j}, p_{k}\right), \\
& S_{2 \mathrm{C}}\left(M, \lambda ; p_{j}, p_{k}\right)=S_{2 \mathrm{C}}\left(\lambda, M ; p_{k}, p_{j}\right) .
\end{aligned}
$$

The relation

$$
S_{3 \mathrm{~L}}\left(M_{a}, M_{b} ; p_{j}, p_{k}, p_{l}\right)+S_{3 \mathrm{~L}}\left(M_{b}, M_{a} ; p_{j}, p_{l}, p_{k}\right) \stackrel{\mathrm{NLL}_{\mathrm{a}}}{=} S\left(M_{a} ; p_{j}, p_{k}\right) S\left(M_{b} ; p_{j}, p_{l}\right)
$$

\footnotetext{
${ }^{7}$ The relations are actually valid for the $\theta$-function representations given in App. B] as well, which contain also NNLL.
} 
permits to simplify combinations of 3-leg ladder integrals for all cases (2.11). Furthermore

$$
\sum_{\pi(j, k, l)} \operatorname{sgn}(\pi(j, k, l)) S_{3 \mathrm{~L}}\left(M, M ; p_{\pi_{l}}, p_{\pi_{k}}, p_{\pi_{j}}\right) \stackrel{\mathrm{NLL}_{\mathrm{a}}}{=} 0,
$$

where the sum runs over all permutations $\pi(j, k, l)$ of $j, k, l$, and $\operatorname{sgn}(\pi(j, k, l))$ is the sign of the permutation. For the 3-leg Yang-Mills diagram we have

$$
S_{3 \mathrm{Y}}\left(M, M, M ; p_{j}, p_{k}, p_{l}\right) \stackrel{\mathrm{NLL}}{=} 0,
$$

as is evident from the totally antisymmetric property in the external momenta $p_{j}, p_{k}$ and $p_{l}$. In presence of a photon, this feature is absent owing to the mass gap $\lambda \ll M$, and we find instead

$$
S_{3 \mathrm{Y}}\left(M, \lambda, M ; p_{j}, p_{k}, p_{l}\right) \stackrel{\mathrm{NLL} a}{=} \frac{1}{2}\left[\Delta S_{3 \mathrm{~L}}\left(M, \lambda ; p_{j}, p_{l}, p_{k}\right)-\Delta S_{3 \mathrm{~L}}\left(M, \lambda ; p_{l}, p_{j}, p_{k}\right)\right],
$$

where $\Delta S_{3 \mathrm{~L}}$ is the subtracted part of the 3-leg ladder diagram as defined in (2.13). This is related to the subtracted part of the 2-leg crossed ladder diagram by

$$
\Delta S_{3 \mathrm{~L}}\left(M, \lambda ; p_{j}, p_{l}, p_{k}\right) \stackrel{\mathrm{NLL}}{=} \Delta S_{2 \mathrm{C}}\left(M, \lambda ; p_{j}, p_{k}\right) .
$$

\section{References}

[1] S. Haywood, P.R. Hobson, W. Hollik, Z. Kunszt et al., hep-ph/0003275, in Standard Model Physics (and more) at the LHC, eds. G. Altarelli and M.L. Mangano, (CERN2000-004, Genève, 2000) p. 117.

[2] E. Accomando et al. [ECFA/DESY LC Physics Working Group Collaboration], Phys. Rept. 299 (1998) 1 [hep-ph/9705442].

[3] J. A. Aguilar-Saavedra et al., TESLA Technical Design Report Part III: Physics at an $\mathrm{e}^{+} \mathrm{e}^{-}$Linear Collider, hep-ph/0106315.

[4] T. Abe et al. [American Linear Collider Working Group Collaboration], in Proc. of the APS/DPF/DPB Summer Study on the Future of Particle Physics (Snowmass 2001), ed. R. Davidson and C. Quigg, SLAC-R-570, Resource book for Snowmass 2001 hep-ex/0106055, hep-ex/0106056, hep-ex/0106057, hep-ex/0106058.

[5] K. Abe et al. [ACFA Linear Collider Working Group Collaboration], ACFA Linear Collider Working Group report [hep-ph/0109166].

[6] M. Kuroda, G. Moultaka and D. Schildknecht, Nucl. Phys. B 350 (1991) 25;

G. Degrassi and A. Sirlin, Phys. Rev. D 46 (1992) 3104;

A. Denner, S. Dittmaier and R. Schuster, Nucl. Phys. B 452 (1995) 80;

A. Denner, S. Dittmaier and T. Hahn, Phys. Rev. D 56 (1997) 117;

A. Denner and T. Hahn, Nucl. Phys. B 525 (1998) 27;

M. Beccaria et al., Phys. Rev. D 58 (1998) 093014 [hep-ph/9805250]. 
[7] V. V. Sudakov, Sov. Phys. JETP 3 (1956) 65 [Zh. Eksp. Teor. Fiz. 30 (1956) 87].

[8] M. Ciafaloni, P. Ciafaloni and D. Comelli, Phys. Rev. Lett. 84 (2000) 4810 [hepph/0001142]; Nucl. Phys. B 589 (2000) 359 [hep-ph/0004071]; Phys. Lett. B 501 (2001) 216 [hep-ph/0007096]; Phys. Rev. Lett. 87 (2001) 211802 [hep-ph/0103315]; Nucl. Phys. B 613 (2001) 382 [hep-ph/0103316].

[9] M. Melles, Phys. Rept. 375 (2003) 219 [hep-ph/0104232].

[10] A. Denner, in Proc. of the International Europhysics Conference on High-Energy Physics (HEP 2001) [hep-ph/0110155].

[11] W. Beenakker et al., Nucl. Phys. B 410 (1993) 245; Phys. Lett. B 317 (1993) 622.

[12] P. Ciafaloni and D. Comelli, Phys. Lett. B 446 (1999) 278 [hep-ph/9809321].

[13] M. Beccaria et al., Phys. Rev. D 61 (2000) 073005 [hep-ph/9906319]; Phys. Rev. D 61 (2000) 011301 [hep-ph/9907389];

M. Beccaria, F. M. Renard and C. Verzegnassi, Phys. Rev. D 63 (2001) 053013 [hepph/0010205]; Phys. Rev. D 63 (2001) 095010 [hep-ph/0007224]; hep-ph/0203254;

M. Beccaria et al., Phys. Rev. D 64 (2001) 053016 [hep-ph/0104245].

[14] M. Beccaria, F. M. Renard and C. Verzegnassi, Phys. Rev. D 64 (2001) 073008 [hep-ph/0103335].

[15] J. Layssac and F. M. Renard, Phys. Rev. D 64 (2001) 053018 [hep-ph/0104205];

G. J. Gounaris, J. Layssac and F. M. Renard, hep-ph/0207273.

[16] A. Denner and S. Pozzorini, Eur. Phys. J. C 18 (2001) 461 [hep-ph/0010201]; Eur. Phys. J. C 21 (2001) 63 [hep-ph/0104127].

[17] S. Pozzorini, doctoral thesis, Universität Zürich, 2001, hep-ph/0201077.

[18] E. Accomando, A. Denner and S. Pozzorini, Phys. Rev. D 65 (2002) 073003 [hep$\mathrm{ph} / 0110114]$.

[19] J. H. Kühn, A. A. Penin and V. A. Smirnov, Eur. Phys. J. C 17 (2000) 97 [hep$\mathrm{ph} / 9912503]$.

[20] V. S. Fadin et al., Phys. Rev. D 61 (2000) 094002 [hep-ph/9910338].

[21] J. H. Kühn, S. Moch, A. A. Penin and V. A. Smirnov, Nucl. Phys. B 616 (2001) 286 [hep-ph/0106298].

[22] M. Melles, Phys. Rev. D 63 (2001) 034003 [hep-ph/0004056]; Phys. Rev. D 64 (2001) 014011 [hep-ph/0012157]; Phys. Rev. D 64 (2001) 054003 [hep-ph/0102097].

[23] M. Melles, Eur. Phys. J. C 24 (2002) 193 [hep-ph/0108221].

[24] M. Melles, Phys. Lett. B 495 (2000) 81 [hep-ph/0006077]. 
[25] M. Hori, H. Kawamura and J. Kodaira, Phys. Lett. B 491 (2000) 275 [hep$\mathrm{ph} / 0007329]$.

[26] W. Beenakker and A. Werthenbach, Phys. Lett. B 489 (2000) 148 [hep-ph/0005316]; Nucl. Phys. B 630 (2002) 3 [hep-ph/0112030].

[27] K. Hepp, Commun. Math. Phys. 2 (1966) 301.

[28] M. Roth and A. Denner, Nucl. Phys. B 479 (1996) 495 [hep-ph/9605420].

[29] T. Binoth and G. Heinrich, Nucl. Phys. B 585 (2000) 741 [hep-ph//0004013].

[30] J.M. Cornwall, D.N. Levin and G. Tiktopoulos, Phys. Rev. D 10 (1974) 1145;

G.J. Gounaris, R. Kögerler and H. Neufeld, Phys. Rev. D 34 (1986) 3257.

[31] M. Beccaria et al., Phys. Rev. D 65 (2002) 093007 [hep-ph//0112273];

M. Beccaria et al., hep-ph/0210283;

M. Melles, hep-ph/0211104.

[32] V. B. Berestetskii, E.M. Lifshitz, and L.P. Pitaevskii, Quantum Electrodynamics, Course of Theoretical Physics, Vol. 4, (Oxford, Pergamon Press, 1982).

[33] A. Denner and S. Pozzorini, in preparation. 